\title{
Examples of finite-dimensional rank 2 Nichols algebras of diagonal type
}

\author{
I. Heckenberger
}

\begin{abstract}
Nichols algebras naturally appear in the theory of quantized enveloping algebras of Kac-Moody algebras and in the classification of finite-dimensional pointed Hopf algebras. Assuming that the base field has characteristic zero a list of finite-dimensional rank 2 Nichols algebras of diagonal type is given. Each of them is described in terms of generators and relations. Kharchenko's restricted Poincaré-Birkhoff-Witt basis is visualized using full binary trees.
\end{abstract}

\section{Introduction}

The classification of finite-dimensional Hopf algebras obtained a significant new branch with the series of papers of Andruskiewitsch and Schneider (see the survey [AS02] and the references therein) about pointed Hopf algebras. Their 'lifting method' to classify pointed Hopf algebras led immediately to novel examples and very rich new structures. The study of Nichols algebras is part of this project, and the aim of the present paper is to describe a big class of rank 2 Nichols algebras of diagonal type in terms of generators and relations; see Theorem 7.1. In [Hec04] it is proven that in a natural setting (the finiteness of the set of Poincaré-Birkhoff-Witt (PBW) generators given by Kharchenko in [Kha99]) the set of examples is exactly the one studied in the present paper. In particular, all finite-dimensional rank 2 Nichols algebras of diagonal type can be obtained from Table 1 (see $\S 7$ ). Additionally, the list presented here contains multiparameter versions of the upper triangular part of quantized enveloping algebras of simple (super) Lie algebras and several new exceptional examples.

The roots of the theory of Nichols algebras can be found in the works of Nichols [Nic78]. Nichols algebras are studied most intensively as part of Hopf algebra theory; see e.g. [AG99, And04, AS98, AS02, Gra00b, Kha99, Ufe04] and the references therein. They can also be seen as a generalization of exterior algebras; see [Sch96, Wor89].

The importance of Nichols algebras stems from the fact that the graded Hopf algebra $H$ associated to the coradical filtration of a finite-dimensional pointed Hopf algebra over a field $k$ can be written as the Radford biproduct $H \cong R \# k G$, where $G$ is a finite group (the group of group-like elements of $H$ ) and $R$ is a braided Hopf algebra. In many known cases $R$ is generated as an algebra by the vector space $V$ of its primitive elements and is then denoted by $\mathcal{B}(V)$ and is called a Nichols algebra. Schauenburg [Sch96] proved that $\mathcal{B}(V)$ is uniquely determined by $V$ as a Yetter-Drinfel'd module.

One of the most remarkable properties of Nichols algebras is their relation to root systems of semisimple Lie algebras $[\mathrm{AS} 02, \S 4]$. More explicitly, to any Cartan matrix and any nonzero $q \in k$ one can associate a Yetter-Drinfel'd module $V$ such that the braiding of $V$ is given in terms of $q$ and the matrix $C$. In general the Nichols algebra $\mathcal{B}(V)$ associated to $V$ is finite dimensional if and only if $C$ is of finite type. However there exist finite-dimensional Nichols algebras which are

Received 27 January 2006, accepted in final form 16 June 2006.

2000 Mathematics Subject Classification 17B37, 16W35.

Keywords: Hopf algebra, Lyndon words, full binary tree.

This journal is (C) Foundation Compositio Mathematica 2007. 


\section{HECKENBERGER}

not of Cartan type. To these exotic algebras one still can associate a (slightly more complicated) 'root system' and 'Dynkin diagram', and one expects that it encodes the full information about the relations of the algebra. In fact, all known finite-dimensional Nichols algebras $\mathcal{B}(V)$ admit a restricted PBW basis labelled by Lyndon words (see e.g. [Kha99, Ros98]), and the degrees of these elements can be interpreted as the set of positive roots of $\mathcal{B}(V)$.

If the action and coaction of $k G$ on $V$ are simultaneously diagonalizable then $V$ is said to be of diagonal type. Currently there exist several classification results in this case; see [AS00, Ros98]. They tell that under some assumptions on $G$ the finiteness of the (Gel'fand-Kirillov) dimension of $\mathcal{B}(V)$ implies that $V$ is related to a symmetrizable Cartan matrix of finite type. Further there exists a list of examples which do not fit into the above classification scheme [AS02]. Andruskiewitsch stated in [And02] the following question.

Question 5.40. Given a braided vector space $V$ of diagonal type and dimension 2, decide when $\mathcal{B}(V)$ is finite dimensional. If so, compute $\operatorname{dim} \mathcal{B}(V)$, and give a 'nice' presentation by generators and relations.

The first part of Question 5.40 of Andruskiewitsch is addressed in [Hec06] and [Hec04]. The aim of the present paper is to give an answer to the second part of Question 5.40 for a large class of rank 2 Nichols algebras. To all of these algebras one can associate on the one hand a generalized Dynkin diagram and on the other hand one of 22 full binary trees. The generalized Dynkin diagram encodes the most essential information of the structure constants of $V$. The full binary tree allows one to read off a generating set of relations and a restricted Poincaré-Birkhoff-Witt basis of $\mathcal{B}(V)$. From this one can also compute the dimension of $\mathcal{B}(V)$. The full binary tree can be considered as an analogue of the root system of a rank 2 semisimple Lie algebra. Indeed, if the braiding of $V$ is of Cartan type, then the set $N_{2}(T)$ of those nodes of the full binary tree $T$ which have two children corresponds to the set of nonsimple positive roots of the Lie algebra. In general, the edges of $T$ and the set of nodes having no children correspond to relations of the Nichols algebra.

The list of (all known and) new examples is contained in Theorem 7.1. Their construction uses several ideas. The computational part relies heavily on the fact that there exists an action of $\mathcal{B}\left(V^{*}\right) \# k G$ on $\mathcal{B}(V)$; see Lemma 4.1 and Corollary 4.1. This already seems to be known and there exist various forms of it in the literature, usually as some bilinear pairing [Lus93] or as quantum differential operators [Gra00a]. The theoretical part is based on the one side on an old result of Stern [Ste58] on some special sequences of pairs of integer numbers. This theory is part of graph theory and is contained also in the modern literature [CLR90]. An adapted version of it is described in $\S 6$. On the other hand deep results of Kharchenko [Kha99] on the structure of certain Hopf algebras are used. They imply that any Nichols algebra of diagonal type and of rank $d$ has a Poincaré-Birkhoff-Witt basis which corresponds to a subset of all Lyndon words of an alphabet with $d$ letters. Kharchenko also proves very strong restrictions on the generating set of relations of such a Nichols algebra. Finally, using the special situation when $V$ has rank 2 one can relate full binary trees $T$ and Nichols algebras $\mathcal{B}(V)$ such that nodes of $T$ correspond to PBW generators and relations of $\mathcal{B}(V)$. This is done in $\S 6$, see Lemma 6.2. In order to check correlations between $T$ and $V$ one still has to perform tedious computations but as an advantage one can eventually avoid the use of computer algebra programs.

If not stated otherwise the definitions and notation follow [AS98]. Throughout this paper $k$ denotes a field of characteristic zero and tensor products $\otimes$ are taken over this field. For Hopf algebras the coproduct and the antipode are denoted by $\Delta$ and $\kappa$, respectively. We use the Sweedler notation $\Delta(a)=a_{(1)} \otimes a_{(2)}$ for elements $a$ of a Hopf algebra. The set of natural numbers not including 0 is denoted by $\mathbb{N}$ and we write $\mathbb{N}_{0}$ for the set $\mathbb{N} \cup\{0\}$. Define $k^{*}:=k \backslash\{0\}$ and $R_{r}:=$ $\left\{q \in k^{*} \mid q^{r}=1, q^{m} \neq 1\right.$ for all $\left.m \in \mathbb{N}, m<r\right\}$ for an arbitrary $r \in \mathbb{N}$. 


\section{RANK 2 NiChOlS ALGEBRAS OF DiAGONAL TYPE}

\section{Yetter-Drinfel'd modules}

Let $k$ be a field, $G$ a group, and $V$ a finite-dimensional vector space over $k$. Then $V$ is called a Yetter-Drinfel'd module over the group algebra $k G$ if for all $g \in G$ there exist subspaces $V_{g} \subset V$ such that $V=\bigoplus_{g \in G} V_{g}$, and $V$ is a left $G$-module with left action . : $k G \otimes V \rightarrow V$ which satisfies the condition $g \cdot v \in V_{g h g^{-1}}$ for all $g, h \in G$ and $v \in V_{h}$. In Hopf algebraic terminology one says that $V$ is a left $k G$-module and a left $k G$-comodule with left coaction $\delta: V \rightarrow k G \otimes V$, and the condition

$$
\delta(a . v)=a_{(1)} v_{(-1)} \kappa\left(a_{(3)}\right) \otimes a_{(2)} \cdot v_{(0)}
$$

holds for all $a \in k G$ and $v \in V$, where $\delta(v)=v_{(-1)} \otimes v_{(0)}$.

Any Yetter-Drinfel'd module has a braiding $\sigma \in \operatorname{Aut}_{k}(V \otimes V)$ defined by the formula $\sigma(v \otimes w)=$ $g . w \otimes v \in V_{g h g^{-1}} \otimes V_{g}$ for all $v \in V_{g}$ and $w \in V_{h}$, and hence $\sigma$ satisfies the braid relation

$$
(\sigma \otimes \mathrm{id})(\mathrm{id} \otimes \sigma)(\sigma \otimes \mathrm{id})=(\mathrm{id} \otimes \sigma)(\sigma \otimes \mathrm{id})(\mathrm{id} \otimes \sigma)
$$

on $V \otimes V \otimes V$. More generally, the braiding $\sigma$ and its inverse are defined by the rules

$$
\sigma(v \otimes w)=\left(v_{(-1)} \cdot w\right) \otimes v_{(0)}, \quad \sigma^{-1}(v \otimes w)=w_{(0)} \otimes\left(\kappa^{-1}\left(w_{(-1)}\right) \cdot v\right)
$$

for all $v, w \in V$. Note that Yetter-Drinfel'd module structures on $V$ with different groups can give the same braiding $\sigma$ and not all braidings of $V$ are induced by groups as described above. A pair $(V, \sigma)$ with a finite-dimensional vector space $V$ and an invertible braiding is called a braided vector space.

If $G$ is an abelian group, then for all $g \in G$ the space $V_{g}$ is invariant under the action of $G$. Moreover, if $G$ is finite and $k$ is an algebraically closed field of characteristic zero then for all $g \in G$ the subspace $V_{g}$ decomposes into the direct sum of one-dimensional $G$-modules. Direct sums of onedimensional Yetter-Drinfel'd modules over $k G$ are called of diagonal type [And02, Definition 5.8]. In this case the braiding $\sigma$ has a particularly simple form. Assume that $V=\bigoplus_{i=1}^{n} V_{i}$ and each $V_{i}$ is a one-dimensional Yetter-Drinfel'd module over $k G$. Then there are unique $g_{i} \in G$ such that $V_{i} \subset V_{g_{i}}$, and numbers $q_{i j} \in k^{*}=k \backslash\{0\}$ such that $\left.g_{i}\right|_{V_{j}}=q_{i j}$ id. One obtains that $\sigma(v \otimes w)=q_{i j} w \otimes v$ for all $v \in V_{i}$ and $w \in V_{j}$. One says that $\sigma$ is of diagonal type. All braided vector spaces of diagonal type can be obtained from Yetter-Drinfel'd modules over abelian groups as described above.

\section{Definitions and notation}

From now on let $k$ be a field of characteristic zero. A bicharacter on a group $(H, \cdot)$ (with values in $\left.k^{*}\right)$ is a map $\chi: H \times H \rightarrow k^{*}$ satisfying the equations

$$
\begin{gathered}
\chi(1, a)=\chi(a, 1)=1 \\
\chi(a \cdot b, c)=\chi(a, c) \chi(b, c), \quad \chi(a, b \cdot c)=\chi(a, b) \chi(a, c)
\end{gathered}
$$

for all $a, b, c \in H$. Then $\chi^{\mathrm{op}}: H \times H \rightarrow k^{*}, \chi^{\mathrm{op}}(a, b):=\chi(b, a)$ is again a bicharacter, and the notation $\chi \chi^{\mathrm{op}}(a, b):=\chi(a, b) \chi(b, a)$ will be used for $a, b \in H$.

Let $d \in \mathbb{N}$ and $\chi$ a bicharacter on $\mathbb{Z}^{d}$. Choose a basis $E=\left\{\mathbf{e}_{i} \mid 1 \leqslant i \leqslant d\right\}$ of $\mathbb{Z}^{d}$. The numbers $q_{i j}:=\chi\left(\mathbf{e}_{i}, \mathbf{e}_{j}\right)$ are called the structure constants of $\chi$ with respect to $E$. For given $E$ they determine $\chi$ uniquely.

Definition 3.1. Let $E$ be a basis of $\mathbb{Z}^{d}$, and $\chi$ a bicharacter on $\mathbb{Z}^{d}$. Let $q_{i j}, i, j \in I:=\{1, \ldots, d\}$, denote the structure constants of $\chi$ with respect to $E$. The generalized Dynkin diagram of the pair $(\chi, E)$ is a (nondirected) graph $\mathcal{D}_{\chi, E}$ with the following properties.

(i) There is a bijective map $\phi$ from $I$ to the set of vertices of $\mathcal{D}_{\chi, E}$.

(ii) For all $i \in I$ the vertex $\phi(i)$ is labelled by $q_{i i}$. 


\section{HECKENBERGER}

(iii) For all $i, j \in I$ the number $n_{i j}$ of edges between $\phi(i)$ and $\phi(j)$ is either 0 or 1 . If $i=j$ or $q_{i j} q_{j i}=1$ then $n_{i j}=0$, otherwise $n_{i j}=1$ and the edge is labelled by $q_{i j} q_{j i}$.

Let $(V, \sigma)$ be a $d$-dimensional braided vector space of diagonal type, where $d \in \mathbb{N}$. For the purpose of the present paper the following definition of a Nichols algebra will be the most appropriate one.

Definition 3.2. Let $S_{m} \in \operatorname{End}_{k}\left(V^{\otimes m}\right)$ and $S_{1, j} \in \operatorname{End}_{k}\left(V^{\otimes j+1}\right)$ denote the maps

$$
\begin{gathered}
S_{m}=\prod_{j=1}^{m-1}\left(\mathrm{id}^{\otimes m-j-1} \otimes S_{1, j}\right), \\
S_{1, j}=\mathrm{id}+\sigma_{12}^{-1}+\sigma_{12}^{-1} \sigma_{23}^{-1}+\cdots+\sigma_{12}^{-1} \sigma_{23}^{-1} \cdots \sigma_{j, j+1}^{-1}
\end{gathered}
$$

(in leg notation) for $m \geqslant 2$ and $j \in \mathbb{N}$. Then the subspace $S=\bigoplus_{m=2}^{\infty}$ ker $S_{m}$ of the tensor algebra $V^{\otimes}=\bigoplus_{m=0}^{\infty} V^{\otimes m}$ is a two-sided ideal, and the algebra $\mathcal{B}(V)=V^{\otimes} / S$ is termed the Nichols algebra associated to $(V, \sigma)$. The unique maximal ideal of $\mathcal{B}(V)$ is denoted by $\mathcal{B}(V)^{+}$.

Let $\left\{x_{i} \mid 1 \leqslant i \leqslant d\right\}$ be a basis of $V$ such that there exist numbers $q_{i j} \in k^{*}, i, j \in\{1,2, \ldots, d\}$, with $\sigma\left(x_{i} \otimes x_{j}\right)=q_{i j} x_{j} \otimes x_{i}$. Since $V$ is a braided vector space of diagonal type, such a basis always exists and is called a canonical basis of $V$. For convenience choose an abelian group $G$ (for example $\mathbb{Z}^{d}$ ) and elements $g_{1}, \ldots, g_{d} \in G$ such that the assignments

$$
\delta\left(x_{i}\right)=g_{i} \otimes x_{i}, \quad g_{i} \cdot x_{j}=q_{i j} x_{j}
$$

define a Yetter-Drinfel'd module structure on $V$. Let $V^{*}$ denote the Yetter-Drinfel'd module dual to $V$. More precisely, for $f \in V^{*}$ one has

$$
(h . f)(v)=f(\kappa(h) . v), \quad f_{(0)}(v) f_{(-1)}=f\left(v_{(0)}\right) \kappa^{-1}\left(v_{(-1)}\right) \quad \forall v \in V, h \in k G,
$$

where $\delta(f)=f_{(-1)} \otimes f_{(0)}$. Note that the antipode $\kappa$ of group algebras satisfies $\kappa^{2}=$ id and hence left and right duals of Yetter-Drinfel'd modules coincide. Let $\left\{y_{i} \mid 1 \leqslant i \leqslant d\right\}$ denote the dual basis of $V^{*}$. Then one has

$$
\delta\left(y_{i}\right)=g_{i}^{-1} \otimes y_{i}, \quad g_{i} \cdot y_{j}=q_{i j}^{-1} y_{j}, \quad \sigma\left(y_{i} \otimes y_{j}\right)=q_{i j} y_{j} \otimes y_{i} .
$$

Thus for diagonal braidings the linear map $\iota: V \rightarrow V^{*}, \iota\left(x_{i}\right):=y_{i}$ for $1 \leqslant i \leqslant d$, extends to an algebra isomorphism $\iota: \mathcal{B}(V) \rightarrow \mathcal{B}\left(V^{*}\right)$.

Let $E=\left\{\mathbf{e}_{i} \mid 1 \leqslant i \leqslant d\right\}$ denote a basis of the $\mathbb{Z}$-module $\mathbb{Z}^{d}$. The tensor algebra $V^{\otimes}$ admits a $\mathbb{Z}^{d}$-grading defined by

$$
\operatorname{deg} x_{i}:=\mathbf{e}_{i}, \quad 1 \leqslant i \leqslant d .
$$

The corresponding total degree totdeg is the $\mathbb{Z}$-grading of $V^{\otimes}$ defined by $\operatorname{totdeg}\left(x_{i}\right)=1$ for $1 \leqslant$ $i \leqslant d$. Note that the map $\sigma$ is $\mathbb{Z}^{d}$-graded and hence the Nichols algebra $\mathcal{B}(V)$ inherits the $\mathbb{Z}^{d}$ - and the $\mathbb{Z}$-grading of $V^{\otimes}$. Let $\mathcal{B}(V)_{n}, n \in \mathbb{N}_{0}$, denote the set of homogeneous elements of $\mathcal{B}(V)$ of total degree $n$.

Let $V$ be a Yetter-Drinfel'd module of diagonal type over an abelian group $G$, and let $\left\{x_{1}, \ldots, x_{d}\right\}$ be a basis of $V$ such that the equations in (2) hold for certain $g_{i} \in G$ and $q_{i j} \in k^{*}$. Choose a basis $E=\left\{\mathbf{e}_{1}, \ldots, \mathbf{e}_{d}\right\}$ of $\mathbb{Z}^{d}$. Then there exist a unique group homomorphism $g: \mathbb{Z}^{d} \rightarrow G$ and a unique bicharacter $\chi: \mathbb{Z}^{d} \times \mathbb{Z}^{d} \rightarrow k^{*}$ satisfying

$$
g\left(\mathbf{e}_{i}\right)=g_{i}, \quad \chi\left(\mathbf{e}_{i}, \mathbf{e}_{j}\right)=q_{i j}
$$

for $i, j \in\{1,2, \ldots, d\}$. For notational convenience we will also write $g(x)$ and $\chi\left(x^{\prime}, x^{\prime \prime}\right)$ instead of $g(\operatorname{deg} x)$ and $\chi\left(\operatorname{deg} x^{\prime}, \operatorname{deg} x^{\prime \prime}\right)$ for homogeneous elements $x, x^{\prime}, x^{\prime \prime} \in V^{\otimes}$ and $x, x^{\prime}, x^{\prime \prime} \in \mathcal{B}(V)$. The generalized Dynkin diagram of $(\chi, E)$ will also be called the generalized Dynkin diagram of $V$. 


\section{RANK 2 NiChOlS ALGEBRAS OF DiAGONAL TYPE}

On the other hand, if $E=\left\{\mathbf{e}_{1}, \ldots, \mathbf{e}_{d}\right\}$ is a basis of $\mathbb{Z}^{d}$ and $\chi$ is a bicharacter on $\mathbb{Z}^{d}$ then there exists a unique (up to isomorphism) braided vector space $(V, \sigma)$ such that the equations $\sigma\left(x_{i} \otimes x_{j}\right)=\chi\left(\mathbf{e}_{i}, \mathbf{e}_{j}\right) x_{j} \otimes x_{i}$, where $i, j \in\{1,2, \ldots, d\}$, hold with respect to a canonical basis $\left\{x_{1}, \ldots, x_{d}\right\}$ of $V$. One says that $(V, \sigma)$ is the braided vector space associated to the pair $(\chi, E)$.

\section{Duality of Nichols algebras}

Now we are going to give a slightly modified version (see Lemma 4.1) of Lusztig's bilinear form [Lus93]; cf. [AS02]. It is closely related to differential operators on $\mathcal{B}(V)$ defined in [AS02, Gra00a, Gra00b].

By Definition 3.2 there exists a bilinear map $\langle\cdot, \cdot\rangle: V^{*} \times \mathcal{B}(V) \rightarrow \mathcal{B}(V)$ such that $\langle f, 1\rangle:=0$ and

$$
\langle f, \rho\rangle:=\sum_{i} f\left(a_{i}\right) \rho_{i}, \quad \text { where } S_{1, m-1}(\rho)=\sum_{i} a_{i} \otimes \rho_{i} \in V \otimes V^{\otimes m-1}
$$

for $m>0$ and $\rho \in V^{\otimes m}$. Since $(f \otimes \mathrm{id}) \sigma^{-1}(v \otimes w)=\left(f_{(-1)} \cdot v\right) f_{(0)}(w)$ for all $v, w \in V$ and $f \in V^{*}$ the map $\langle\cdot, \cdot\rangle$ has the property

$$
\left\langle f, \rho \rho^{\prime}\right\rangle=\langle f, \rho\rangle \rho^{\prime}+\left(f_{(-1)} \cdot \rho\right)\left\langle f_{(0)}, \rho^{\prime}\right\rangle
$$

for all $f \in V^{*}$ and $\rho, \rho^{\prime} \in \mathcal{B}(V)$. Note that $\rho=0$ in $\mathcal{B}(V)^{+}$if and only if $\langle f, \rho\rangle=0$ for all $f \in V^{*}$.

Let $\mathcal{B}\left(V^{*}\right) \# k G$ denote the set $\mathcal{B}\left(V^{*}\right) \otimes k G$ with the product

$$
\left(f^{\prime} \otimes g^{\prime}\right)\left(f^{\prime \prime} \otimes g^{\prime \prime}\right):=f^{\prime}\left(g^{\prime} \cdot f^{\prime \prime}\right) \otimes g^{\prime} g^{\prime \prime}
$$

for all $f^{\prime}, f^{\prime \prime} \in \mathcal{B}\left(V^{*}\right)$ and $g^{\prime}, g^{\prime \prime} \in G$. Then $\mathcal{B}\left(V^{*}\right) \# k G$ becomes a Hopf algebra with coproduct

$$
\Delta(f)=f \otimes 1+\delta(f), \quad \Delta(g)=g \otimes g, \quad f \in V^{*}, g \in G .
$$

Lemma 4.1. There exists a unique bilinear map $\langle\cdot, \cdot\rangle:\left(\mathcal{B}\left(V^{*}\right) \# k G\right) \times \mathcal{B}(V) \rightarrow \mathcal{B}(V)$ satisfying $\langle f, v\rangle=f(v)$ for $f \in V^{*}, v \in V$, and

$$
\begin{gathered}
\left\langle f, \rho \rho^{\prime}\right\rangle=\langle f, \rho\rangle \rho^{\prime}+\left(f_{(-1)} \cdot \rho\right)\left\langle f_{(0)}, \rho^{\prime}\right\rangle, \quad\langle g, \rho\rangle=g . \rho, \\
\left\langle h_{1} h_{2}, \rho\right\rangle=\left\langle h_{1},\left\langle h_{2}, \rho\right\rangle\right\rangle
\end{gathered}
$$

for all $f \in V^{*}, g \in k G, h_{1}, h_{2} \in \mathcal{B}\left(V^{*}\right) \# k G$, and $\rho, \rho^{\prime} \in \mathcal{B}(V)$.

Since $\Delta(f)=f \otimes 1+\delta(f)$ for $f \in V$ and $\Delta(g)=g \otimes g$ for $g \in G$ the following corollary is an immediate consequence of Lemma 4.1.

Corollary 4.1. For any $f \in \mathcal{B}\left(V^{*}\right) \# k G$ and $\rho, \rho^{\prime} \in \mathcal{B}(V)$ the formula $\left\langle f, \rho \rho^{\prime}\right\rangle=\left\langle f_{(1)}, \rho\right\rangle\left\langle f_{(2)}, \rho^{\prime}\right\rangle$ holds where $\Delta(f)=f_{(1)} \otimes f_{(2)}$.

Proof of Lemma 4.1. It is clear that the restriction of $\langle\cdot, \cdot\rangle$ to $V^{*} \times \mathcal{B}(V)$ has to be the map $\langle\cdot, \cdot\rangle$ introduced above. Therefore the uniqueness assertion immediately follows. Further, the equation

$$
\left\langle g^{-1} . f, \rho\right\rangle=g^{-1} \cdot\langle f, g . \rho\rangle, \quad g \in G, f \in V^{*}, \rho \in \mathcal{B}(V),
$$

implies that there exists at least a map $\langle\cdot, \cdot\rangle:\left(\bigoplus_{m=0}^{\infty} V^{* \otimes m} \# k G\right) \times \mathcal{B}(V) \rightarrow \mathcal{B}(V)$ satisfying the above equations.

For $1 \leqslant l \leqslant m$ there exist linear maps $S_{l, m-l} \in \operatorname{End}_{k}\left(V^{\otimes m}\right)$ such that the equation

$$
\prod_{j=1}^{l}\left(\mathrm{id}^{\otimes l-j} \otimes S_{1, m-l+j-1}\right)=\left(S_{l} \otimes \mathrm{id}^{\otimes m-l}\right) S_{l, m-l}
$$

holds. Thus for $l, m \in \mathbb{N}_{0}, l \leqslant m, f_{i} \in V^{*}, 1 \leqslant i \leqslant l$, and $\rho \in V^{\otimes m}$ one has

$$
\left\langle f_{1},\left\langle f_{2}, \cdots\left\langle f_{l}, \rho\right\rangle\right\rangle\right\rangle=\left(f_{l} \otimes f_{l-1} \otimes \cdots f_{1} \otimes \mathrm{id}^{m-l}\right)\left(\left(S_{l} \otimes \mathrm{id}^{m-l}\right) S_{l, m-l}(\rho)\right) .
$$




\section{HECKENBERGER}

Moreover, direct computation shows that

$$
(f \otimes g)\left(\sigma^{-1}(v \otimes w)\right)=\sigma^{-1}(g \otimes f)(w \otimes v)
$$

for $f, g \in V^{*}$ and $v, w \in V$. Therefore $\left\langle\operatorname{ker} S_{l}^{*}, \mathcal{B}(V)\right\rangle=0$ where the map $S_{l}^{*} \in \operatorname{End}_{k}\left(V^{* \otimes l}\right)$ is defined analogously to $S_{l}$.

The skew-differential operators $\left\langle y_{i}, \cdot\right\rangle \in \operatorname{End}_{k}(\mathcal{B}(V))$ give immediately the following information about $\mathcal{B}(V)$. For $\rho \in \mathcal{B}(V)$ define $\left(\operatorname{ad}_{\sigma} x_{i}\right)(\rho):=x_{i} \rho-\left(g_{i} . \rho\right) x_{i}$. Moreover, for $n \in \mathbb{N}_{0}$ and $q \in k^{*}$ set $(n)_{q}:=\sum_{i=0}^{n-1} q^{i}$ and $(n)_{q}^{!}:=\prod_{i=1}^{n}(i)_{q}$.

LEMma 4.2 [AS02, Lemma 3.7]. One has the following.

(i) One has $x_{i}^{n}=0$ if and only if $q_{i i} \in R_{r}$, where $1<r \leqslant n$.

(ii) If $i \neq j$ then $\left(\operatorname{ad}_{\sigma} x_{i}\right)^{r}\left(x_{j}\right)=0$ if and only if $(r)_{q_{i i}}^{!} \prod_{l=0}^{r-1}\left(q_{i i}^{l} q_{i j} q_{j i}-1\right)=0$.

Proof. By induction one obtains the formulas

$$
\begin{aligned}
\left\langle y_{j}, x_{j}^{r}\right\rangle & =(r)_{q_{j j}^{-1}} x_{j}^{r-1}, \quad\left\langle y_{l}, x_{j}^{r}\right\rangle=0, \\
\left\langle y_{j},\left(\operatorname{ad}_{\sigma} x_{i}\right)^{r}\left(x_{j}\right)\right\rangle & =\prod_{l=0}^{r-1}\left(q_{j i}^{-1}-q_{i i}^{l} q_{i j}\right) x_{i}^{r}, \quad\left\langle y_{l},\left(\operatorname{ad}_{\sigma} x_{i}\right)^{r}\left(x_{j}\right)\right\rangle=0
\end{aligned}
$$

for all $l \neq j$. This implies the claim.

\section{PBW bases of Nichols algebras of diagonal type}

In [Kha99] Kharchenko proves that certain Hopf algebras 'of diagonal type' have a PoincaréBirkhoff-Witt basis. He also gives very useful information on the relations of the algebra. It is worth mentioning that (as was shown by Ufer in [Ufe04]) such results hold in a more general context, namely if the diagonal braiding is generalized to a triagonal one. In order to prove Theorem 7.1 results from [Lot83] and [Kha99] are recalled and adapted to our conventions. The symbol $>$ for the lexicographic order in [Kha99] is replaced by $<$.

Set $X:=\left\{\alpha_{1}, \ldots, \alpha_{d}\right\}$ and consider the total order $<$ on $X$ given by $\alpha_{i}<\alpha_{j}$ for $i<j$. Let $\mathbb{X}$ and $\mathbb{X}^{+}$denote the set of words and nonempty words, respectively, in the letters of $X$. Then $<$ induces the lexicographic order on $\mathbb{X}: u, v \in \mathbb{X}$ satisfy $u<v$ if and only if either $v=u w$ for some $w \in \mathbb{X}^{+}$or there exist $u_{1}, u_{2}, u_{3} \in \mathbb{X}$ and $i<j$ such that $u=u_{1} \alpha_{i} u_{2}$ and $v=u_{1} \alpha_{j} u_{3}$. For $u, v \in \mathbb{X}$ we write $u \leqslant v$ if $u=v$ or $u<v$. The length of a word $u$, i.e. the number of its letters, is denoted by $|u|$. A word $u \in \mathbb{X}^{+}$is called a Lyndon word if for any decomposition $u=v w$ with $v, w \in \mathbb{X}^{+}$ the relation $v w<w v$ holds.

Proposition 5.1. One has the following.

(i) A word $u \in \mathbb{X}^{+}$is a Lyndon word if and only if $u=v w$ with $v, w \in \mathbb{X}^{+}$implies $u<w$ (see [Lot83, Proposition 5.1.2]).

(ii) $A$ word $u \in \mathbb{X}^{+}$is a Lyndon word if and only if either $u \in X$ or there exist Lyndon words $v, w \in \mathbb{X}$ such that $v<w$ and $u=v w$ (see [Lot83, Proposition 5.1.3]).

Any word $u \in \mathbb{X}$ has a unique decomposition into the product of a nonincreasing sequence of Lyndon words [Lot83, Theorem 5.1.5]. Further, any Lyndon word $u \notin X$ has a decomposition into the product of two Lyndon words $u=v w$ (which then satisfy $v<w$ ) such that $|v|$ is minimal. This is called the Shirshow decomposition of $u$. 


\section{RANK 2 NiChOlS ALGEBRAS OF DiAGONAL TYPE}

Proposition 5.2 [Lot83, Proposition 5.1.4]. Suppose that $u, v, w$ are Lyndon words and $u=v w$. Then $u=v w$ is the Shirshow decomposition of $u$ if and only if $v \in X$ or for the Shirshow decomposition $v=v_{1} v_{2}$ the relation $w \leqslant v_{2}$ holds.

Let $k$ be a field of characteristic zero, $G$ an abelian group, and $V \in{ }_{k G}^{k G} \mathcal{Y D}$ a $d$-dimensional YetterDrinfel'd module of diagonal type. Let $\mathcal{I}$ be an $\mathbb{N}_{0}^{d}$-graded ideal of $V^{\otimes}, \mathcal{A}$ the $\mathbb{N}_{0}^{d}$-graded algebra $V^{\otimes} / \mathcal{I}$, and $\mathcal{A}_{n}$ the subspace of $\mathcal{A}$ of homogeneous elements of total degree $n$ (where $n \in \mathbb{N}_{0}$ ). Let $\mathcal{A}^{+}$denote the unique maximal ideal of $\mathcal{A}$. The following results will be needed for $\mathcal{A}=V^{\otimes}$ and for $\mathcal{A}=\mathcal{B}(V)$.

After fixing a canonical basis $\left\{x_{1}, x_{2}, \ldots, x_{d}\right\}$ of $V$ one can associate to any Lyndon word $u \in \mathbb{X}$ an element $[u] \in \mathcal{A}$ as follows. Set $\left[\alpha_{i}\right]:=x_{d+1-i}$ for $1 \leqslant i \leqslant d$, and $[u]:=[w][v]-\chi([w],[v])[v][w]$ if $u=v w$ is the Shirshow decomposition of $u$. Note that this definition differs from that in [Kha99] by a constant for each Lyndon word $u$. However this is not relevant for the following assertions.

Lemma 5.1 [Kha99, Lemma 3]. If $u, v$ are Lyndon words with $u<v$ then $u^{h}<v$ for any $h>0$.

Lemma 5.2 [Kha99, Lemma 5]. Let $u$ be a Lyndon word with $|u|=m$. Then $[u] \in \mathcal{A}$ is a linear combination of monomials $\left[\alpha_{i_{1}}\right]\left[\alpha_{i_{2}}\right] \cdots\left[\alpha_{i_{m}}\right] \in \mathcal{A}, \alpha_{i_{j}} \in X$, such that $u \leqslant \alpha_{i_{1}} \alpha_{i_{2}} \cdots \alpha_{i_{m}}$.

Lemma 5.3 [Kha99, Lemma 6]. If $u<v \in \mathbb{X}$ are Lyndon words then $[v][u]-\chi([v],[u])[u][v]$ is a linear combination of products $\left[u_{1}\right]\left[u_{2}\right] \cdots\left[u_{i}\right]$ for certain $i \in \mathbb{N}$ and Lyndon words $u_{j}$ with $u<u_{j}<v$ such that $\operatorname{deg}([v][u])=\operatorname{deg}\left(\left[u_{1}\right]\left[u_{2}\right] \cdots\left[u_{i}\right]\right)$ and $u v \leqslant u_{1} u_{2} \cdots u_{i}$.

For a Lyndon word $u \in \mathbb{X}$ define $h_{u}$ as follows. If $m$ is the smallest number such that $[u]^{m}$ can be written as a linear combination of products $\left[u_{1}\right]\left[u_{2}\right] \cdots\left[u_{i}\right], i \in \mathbb{N}$, where $u_{j}$ are Lyndon words with $u<u_{j}$, and either $m=1$ or $\chi([u],[u])$ is a primitive $m$ th root of 1 , then set $h_{u}:=m$. Otherwise put $h_{u}:=\infty$. By Lemma 5.1 the relation $u<u_{1}$ implies that $u^{h_{u}}<u_{1}$. Now since $u^{h_{u}}$ is not the beginning of $u_{1}$ one obtains that $u^{h_{u}}<u_{1} u_{2} \ldots u_{i}$ has to hold as well.

Define $B:=\left\{u \in \mathbb{X} \mid u\right.$ is a Lyndon word, $\left.h_{u}>1\right\}$. For each $u \in B$ let $S(u)_{<}$and $S(u)$ denote the subalgebras of $\mathcal{A}$ generated by the sets $\{[v] \mid v \in B, u<v\}$ and $\{[v] \mid v \in B, u \leqslant v\}$, respectively. Define $S(u)^{+}:=S(u) \cap \mathcal{A}^{+}$and $S(u)_{<}^{+}:=S(u)_{<} \cap \mathcal{A}^{+}$.

Theorem 5.1 [Kha99, Theorem 2]. The set $\left\{\left[u_{1}\right]^{n_{1}}\left[u_{2}\right]^{n_{2}} \cdots\left[u_{i}\right]^{n_{i}} \mid i \in \mathbb{N}_{0}, u_{j} \in B, u_{i}<\cdots<u_{2}<\right.$ $\left.u_{1}, n_{j}<h_{u_{j}} \forall j\right\}$ is a basis of the vector space $\mathcal{A}$.

Corollary 5.1. For any $n \in \mathbb{N}, u \in B$, the sets

$$
\left\{\left[u_{1}\right]^{n_{1}}\left[u_{2}\right]^{n_{2}} \cdots\left[u_{i}\right]^{n_{i}} \mid i \in \mathbb{N}_{0}, u_{j} \in B, u<u_{1}<u_{2}<\cdots<u_{i}, n_{j}<h_{u_{j}} \forall j\right\}
$$

and

$$
\left\{\left[u_{1}\right]^{n_{1}}\left[u_{2}\right]^{n_{2}} \cdots\left[u_{i}\right]^{n_{i}} \mid i \in \mathbb{N}_{0}, u_{j} \in B, u \leqslant u_{1}<u_{2}<\cdots<u_{i}, n_{j}<h_{u_{j}} \forall j\right\}
$$

form a basis of the vector space $S(u)_{<}$and $S(u)$, respectively.

Proof. Since $S(u)_{<}=\bigcup_{v \in B, u<v} S(v)$ it suffices to prove the assertion for $S(u)$. As $\mathcal{A}=\bigoplus_{n=0}^{\infty} \mathcal{A}_{n}$ and $S(u)=\bigoplus_{n=0}^{\infty} S(u) \cap \mathcal{A}_{n}$ the proof can be performed by induction on $n$. Note that for given $n \in \mathbb{N}_{0}$ the set $\mathcal{A}_{n}$ is finite-dimensional and $\{v \in B \mid \operatorname{deg}([v]) \leqslant n\}$ is a finite set. Suppose that $\left\{u_{1}, u_{2}, \ldots, u_{i}\right\}=\{v \in B \mid u<v, \operatorname{deg}([v]) \leqslant n\}$ and $u_{1}<u_{2}<\cdots<u_{i}$. By Lemma 5.3 the elements $[u]^{m}\left[u_{j_{1}}\right]\left[u_{j_{2}}\right] \cdots\left[u_{j_{r}}\right], m<h_{u}, m \operatorname{deg}([u])+\sum_{s=1}^{r} \operatorname{deg}\left(\left[u_{j_{s}}\right]\right)=n$, span $S(u) \cap \mathcal{A}_{n}$. By induction hypothesis the elements $\left[u_{1}\right]^{n_{1}}\left[u_{2}\right]^{n_{2}} \cdots\left[u_{i}\right]^{n_{i}}, n_{j}<h_{u_{j}}$ for all $j$, where $\sum_{j=1}^{i} n_{j} \operatorname{deg}\left(\left[u_{j}\right]\right)=n-$ $m \operatorname{deg}([u]), \operatorname{span} S\left(u_{1}\right) \cap \mathcal{A}_{n-m \operatorname{deg}([u])}$ for all $m>0$. Therefore the elements $[u]^{m}\left[u_{1}\right]^{n_{1}}\left[u_{2}\right]^{n_{2}} \cdots\left[u_{i}\right]^{n_{i}}$, $n_{j}<h_{u_{j}}$ for all $j, m<h_{u}$, where $m \operatorname{deg}([u])+\sum_{j=1}^{i} n_{j} \operatorname{deg}\left(\left[u_{j}\right]\right)=n$, span $S(u) \cap \mathcal{A}_{n}$. Then Theorem 5.1 and a simple dimension argument imply the assertion. 


\section{HECKENBERGER}

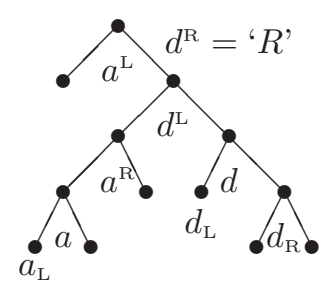

FiguRE 1. Illustration of some definitions.

Later we will need the fact that for $w \in B$ one has

$$
S(w)=\left(S(w) S(w)_{<}^{+}+S(w)[w]^{2}\right) \oplus k[w] \oplus k 1
$$

as graded vector spaces which is one of the consequences of Corollary 5.1 and Lemma 5.3.

Proposition 5.3 [Kha99, Corollary 2]. For a Lyndon word $u$ of length $l$ one has $h_{u}=1$ if and only if $[u]$ can be written as a linear combination of monomials $\left[\alpha_{i_{1}}\right]\left[\alpha_{i_{2}}\right] \cdots\left[\alpha_{i_{l}}\right], \alpha_{i_{j}} \in X$ for all $j$, such that $u<\alpha_{i_{1}} \alpha_{i_{2}} \cdots \alpha_{i_{l}}$.

\section{Types of rank 2 Nichols algebras}

For basic definitions in this section we refer to [CLR90]. Recall that a binary tree $T$ is a (nonempty finite) tree such that each node has at most two children. One says that $T$ is full if each node of $T$ has exactly zero or two children [CLR90]. For examples see Appendix A. For the set of nodes of a full binary tree $T$ which have zero and two children, respectively, we use the symbol $N_{0}(T)$ and $N_{2}(T)$, respectively. Let $\mathbf{r}(T)$ or simply $\mathbf{r}$ denote the root of the binary tree $T$. Further, we write $N(T)=N_{0}(T) \cup N_{2}(T)$ for the set of all nodes of $T$. Let $\left\{{ }^{\prime} L\right.$ ', ' $R$ ' $\}$ be a set with two elements and define $\bar{N}_{2}(T):=N_{2}(T) \cup\left\{{ }^{\prime} L\right.$ ' ' $R$ ' $\}, \bar{N}(T):=N(T) \cup\left\{{ }^{\prime} L\right.$ ' ' $R$ ' $\}$ (disjoint unions).

Definition 6.1. Let $T$ be a full binary tree and $a \in N(T)$. A node $b \in \bar{N}_{2}(T)$ is called the left godfather of $a$, denoted by $b:=a^{\mathrm{L}}$, if one of the following conditions holds:

(i) $a=\mathbf{r}(T)$ and $b={ }^{\prime} L$ ';

(ii) $a$ is the right child of $b$;

(iii) $a$ is the left child of its parent $c$ and $b$ is the left godfather of $c$.

Similarly one defines the right godfather $a^{\mathrm{R}}$ of a by replacing everywhere left by right and vice versa and setting $\mathbf{r}^{\mathrm{R}}:=$ ' $R$ '. If $a \in N_{2}(T)$ then let $a_{\mathrm{L}}$ and $a_{\mathrm{R}}$ denote the left and right child of $a$, respectively.

The above definitions should be demonstrated on an example (see Figure $1^{1}$ ). Note that $a^{\mathrm{L}}=\mathbf{r}$.

Observe that $\cdot{ }^{\mathrm{L}}$ and ${ }^{\mathrm{R}}$ are well-defined maps from $N(T)$ to $\bar{N}_{2}(T)$ and any $a \in N(T)$ is uniquely determined by $a^{\mathrm{L}}$ and $a^{\mathrm{R}}$. Indeed, $a \in N(T), b=a^{\mathrm{L}}$ implies that there is a subset $\left\{a_{1}, a_{2}, \ldots, a_{m}\right\}$ of $N(T)$ such that $m \in \mathbb{N}, a_{m}=a, a_{i+1}$ is the left child of $a_{i}$ for all $i>0$, and either $a_{1}=b_{\mathrm{R}}$ or $a_{1}=\mathbf{r}, b=$ ' $L$ '. Further, $a_{i+1}{ }^{\mathrm{R}}=a_{i}$ for all $i>1$ and $a_{1}{ }^{\mathrm{R}} \notin\left\{a_{1}, a_{2}, \ldots, a_{m}\right\}$. Thus $a$ is uniquely determined by $a^{\mathrm{L}}$ and $a^{\mathrm{R}}$.

\footnotetext{
${ }^{1}$ The labels in Figure 1 belong to the nodes above them.
} 


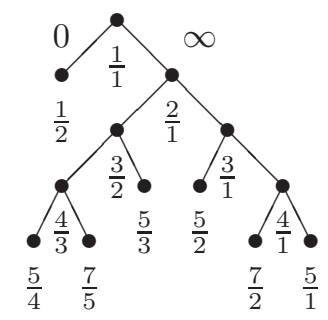

Figure 2. Values of $Q$.

We define functions $\ell^{\mathrm{L}}, \ell^{\mathrm{R}}, \ell_{\mathrm{L}}$, and $\ell_{\mathrm{R}}: N(T) \rightarrow \mathbb{N}$ recursively to denote lengths of certain branches of $T$. Set

$$
\begin{aligned}
\ell^{\mathrm{L}}(a):=\left\{\begin{array}{lll}
\ell^{\mathrm{L}}\left(a^{\mathrm{L}}\right)+1 & \text { if } a^{\mathrm{L}}{ }_{\mathrm{R}}=a, \\
1 & \text { otherwise, }
\end{array}\right. & \ell_{\mathrm{L}}(a):= \begin{cases}1 & \text { if } a \in N_{0}(T), \\
\ell_{\mathrm{L}}\left(a_{\mathrm{L}}\right)+1 & \text { otherwise, }\end{cases} \\
\ell^{\mathrm{R}}(a):= \begin{cases}\ell^{\mathrm{R}}\left(a^{\mathrm{R}}\right)+1 & \text { if } a^{\mathrm{R}}{ }_{\mathrm{L}}=a, \\
1 & \text { otherwise, }\end{cases} & \ell_{\mathrm{R}}(a):= \begin{cases}1 & \text { if } a \in N_{0}(T), \\
\ell_{\mathrm{R}}\left(a_{\mathrm{R}}\right)+1 & \text { otherwise. }\end{cases}
\end{aligned}
$$

In the example of Figure 1 these functions take the following values:

\begin{tabular}{ccccccccc}
\hline & $a$ & $a_{\mathrm{L}}$ & $a^{\mathrm{L}}$ & $a^{\mathrm{R}}$ & $d$ & $d_{\mathrm{L}}$ & $d_{\mathrm{R}}$ & $d^{\mathrm{L}}$ \\
\hline$\ell_{\mathrm{L}}$ & 2 & 1 & 2 & 3 & 2 & 1 & 2 & 4 \\
$\ell_{\mathrm{R}}$ & 2 & 1 & 5 & 2 & 3 & 1 & 2 & 4 \\
$\ell^{\mathrm{L}}$ & 1 & 1 & 1 & 1 & 3 & 1 & 4 & 2 \\
$\ell^{\mathrm{R}}$ & 3 & 4 & 1 & 2 & 1 & 2 & 1 & 1 \\
\hline
\end{tabular}

Any full binary tree $T$ can be identified with a subtree of the infinite Stern-Brocot tree [GKP94, pp. 116-117], see also [Ste58]. This means that there exists a map $\sigma_{T}: \bar{N}(T) \rightarrow \mathbb{Z} \times \mathbb{Z}$ defined recursively by $\sigma_{T}\left({ }^{\prime} L^{\prime}\right):=(0,1), \sigma_{T}\left({ }^{\prime} R^{\prime}\right):=(1,0)$, and $\sigma_{T}(a):=\sigma_{T}\left(a^{\mathrm{L}}\right)+\sigma_{T}\left(a^{\mathrm{R}}\right)$ for any $a \in N(T)$. Note that $\sigma_{T}(a) \in \mathbb{N} \times \mathbb{N}$ for $a \in N(T)$ by the definition of ${ }^{\mathrm{L}}{ }^{\mathrm{and}} \cdot{ }^{\mathrm{R}}$ and since $\sigma_{T}(\mathbf{r})=(1,1)$. Thus the $\operatorname{map} Q: \bar{N}(T) \rightarrow \mathbb{Q} \cup\{+\infty\}$,

$$
Q(a):= \begin{cases}r / s & \text { if } \sigma_{T}(a)=(r, s), s \neq 0, \\ +\infty & \text { if } a='{ }^{\prime} R,\end{cases}
$$

and the total order $<$ on $\mathbb{Q}$ induce an order $<_{Q}$ on $\bar{N}(T)$ such that for all $a \in N(T)$ the relations ' $L$ ' $<_{Q} a<_{Q}$ ' $R$ ' hold. Figure 2 shows the values of the map $Q$ for the example in Figure 1.

Therefore in this case one has the relations

$$
\begin{gathered}
{ }^{\prime} L '<_{Q} a^{\mathrm{L}}{ }_{\mathrm{L}}<_{Q} a^{\mathrm{L}}<_{Q} a_{\mathrm{L}}<_{Q} a<_{Q} a_{\mathrm{R}}<_{Q} a^{\mathrm{R}}<_{Q} a^{\mathrm{R}}{ }_{\mathrm{R}}<_{Q} \\
\quad<_{Q} d^{\mathrm{L}}<_{Q} d_{\mathrm{L}}<_{Q} d<_{Q} d_{\mathrm{RL}}<_{Q} d_{\mathrm{R}}<_{Q} d_{\mathrm{RR}}<_{Q}{ }^{\prime} R .
\end{gathered}
$$

There is another natural map $\left|\sigma_{T}\right|: \bar{N}(T) \rightarrow \mathbb{Z}$ defined by $\left|\sigma_{T}\right|(a)=r+s$ whenever $\sigma_{T}(a)=(r, s)$. It will be used mainly for inductive proofs.

Proofs for assertions (i)-(iii) of the following lemma can also be found for example in [GKP94].

Lemma 6.1. Let $T$ be a full binary tree and $a, b \in N(T)$.

(i) If $\sigma_{T}(a)=(r, s), \sigma_{T}\left(a^{\mathrm{L}}\right)=\left(r_{1}, s_{1}\right), \sigma_{T}\left(a^{\mathrm{R}}\right)=\left(r_{2}, s_{2}\right)$ then the equations $r s_{1}-r_{1} s=r_{2} s-r s_{2}=$ $r_{2} s_{1}-r_{1} s_{2}=1$ hold.

(ii) The entries of $\sigma_{T}(c), c \in \bar{N}(T)$, are relatively prime integers. 


\section{HECKENBERGER}

(iii) The map $Q: \bar{N}(T) \rightarrow \mathbb{Q} \cup\{+\infty\}$ is injective and hence $<_{Q}$ is a total order on $\bar{N}(T)$.

(iv) One has $a^{\mathrm{L}}<_{Q} a<_{Q} a^{\mathrm{R}}$.

(v) If $a<_{Q} b$ and $\left|\sigma_{T}\right|(a)<\left|\sigma_{T}\right|(b)+\left|\sigma_{T}\right|\left(b^{\mathrm{L}}\right)$ then relation $a \leqslant Q{ }_{Q} b^{\mathrm{L}}$ holds.

(vi) If $a<_{Q} b$ and $\left|\sigma_{T}\right|(b)<\left|\sigma_{T}\right|(a)+\left|\sigma_{T}\right|\left(a^{\mathrm{R}}\right)$ then relation $a^{\mathrm{R}} \leqslant_{Q} b$ holds.

(vii) Relation $a^{\mathrm{L}} \in N(T)$ implies that $a<_{Q} a^{\mathrm{LR}}$.

(viii) Relation $a^{\mathrm{R}} \in N(T)$ implies that $a^{\mathrm{RL}}<_{Q} a$.

(ix) If $a \in N(T), b \in \bar{N}(T),\left|\sigma_{T}\right|(b) \leqslant\left|\sigma_{T}\right|(a)$, and $a^{\mathrm{L}}<_{Q} b<_{Q} a^{\mathrm{R}}$ then one has $b=a$.

(x) If $a, b \in \bar{N}_{2}(T)$ such that $a<_{Q} b$ then there exists $c \in N(T)$ such that $a<_{Q} c<_{Q} b$.

Proof. (i) We prove this by induction on $\left|\sigma_{T}\right|(a)$. If $a=\mathbf{r}$ then $\sigma_{T}(a)=(1,1)$ and the assertion holds. Otherwise let $c \in N(T)$ denote the parent of $a$. If $a$ is the left child of $c$ then $a^{\mathrm{R}}=c$, $a^{\mathrm{L}}=c^{\mathrm{L}}$, and $\sigma_{T}(c)=\left(r_{2}, s_{2}\right)$. Since $\left|\sigma_{T}\right|(c)<\left|\sigma_{T}\right|(a)$ equation $\sigma_{T}\left(c^{\mathrm{L}}\right)=\left(r_{1}, s_{1}\right)$ and induction hypothesis gives $r_{2} s_{1}-r_{1} s_{2}=1$. Thus $r s_{1}-r_{1} s=\left(r_{1}+r_{2}\right) s_{1}-r_{1}\left(s_{1}+s_{2}\right)=1$ and $r_{2} s-r s_{2}=$ $r_{2}\left(s_{1}+s_{2}\right)-\left(r_{1}+r_{2}\right) s_{2}=r_{2} s_{1}-r_{1} s_{2}=1$. If $a=c_{\mathrm{R}}$ then one argues similarly.

(ii) One obtained this from assertion (i) using $\sigma_{T}\left({ }^{\prime} L '\right)=(0,1), \sigma_{T}\left({ }^{\prime} R '\right)=(1,0)$.

(iv) This follows from assertion (i) and the fact that $\sigma_{T}(c) \in \mathbb{N} \times \mathbb{N}$ for $c \in N(T)$.

(v) Suppose to the contrary that $b^{\mathrm{L}}<_{Q} a<_{Q} b$ and $\left|\sigma_{T}\right|(a)<\left|\sigma_{T}\right|(b)+\left|\sigma_{T}\right|\left(b^{\mathrm{L}}\right)$. If $\sigma_{T}(a)=(r, s)$, $\sigma_{T}(b)=\left(r_{2}, s_{2}\right)$, and $\sigma_{T}\left(b^{\mathrm{L}}\right)=\left(r_{1}, s_{1}\right)$ then $r_{2} s_{1}-r_{1} s_{2}=1, r s_{1}-r_{1} s \geqslant 1$ and $r_{2} s-r s_{2} \geqslant 1$. Therefore

$$
\begin{aligned}
& \left(r_{2} s\right) s_{1} \geqslant\left(r s_{1}\right) s_{2}+s_{1} \geqslant r_{1} s s_{2}+s_{2}+s_{1} \Rightarrow s_{1}+s_{2} \leqslant s, \\
& r_{2}\left(r s_{1}\right) \geqslant r_{1}\left(r_{2} s\right)+r_{2} \geqslant r_{1} r s_{2}+r_{1}+r_{2} \Rightarrow r_{1}+r_{2} \leqslant r .
\end{aligned}
$$

This is a contradiction to $r+s=\left|\sigma_{T}\right|(a)<\left|\sigma_{T}\right|(b)+\left|\sigma_{T}\right|\left(b^{\mathrm{L}}\right)=r_{1}+r_{2}+s_{1}+s_{2}$.

(vi) Use arguments as in assertion (v).

(iii) If $Q(a)=Q(b)$ then $\sigma_{T}(a)=\sigma_{T}(b)$ by assertion (ii). Now $b^{\mathrm{L}}<_{Q} a,\left|\sigma_{T}\right|\left(b^{\mathrm{L}}\right)<\left|\sigma_{T}\right|(b)=$ $\left|\sigma_{T}\right|(a)$, and assertion (v) imply that $b^{\mathrm{L}} \leqslant Q a^{\mathrm{L}}$. By symmetry one gets $Q\left(a^{\mathrm{L}}\right)=Q\left(b^{\mathrm{L}}\right)$ and similarly $Q\left(a^{\mathrm{R}}\right)=Q\left(b^{\mathrm{R}}\right)$. Thus using that $a^{\mathrm{L}}$ and $a^{\mathrm{R}}$ determine $a$ uniquely, induction on $\left|\sigma_{T}\right|(a)$ gives the assertion.

(vii) Again we use induction on $\left|\sigma_{T}\right|(a)$. Note that $a \neq \mathbf{r}$ since $a^{\mathrm{L}} \in N(T)$. If $a$ is the left child of its parent $c$ then $a^{\mathrm{L}}=c^{\mathrm{L}}$ and $a^{\mathrm{R}}=c$. By induction hypothesis $c<_{Q} c^{\mathrm{LR}}$ and, hence, assertion (iv) implies that $a<_{Q} a^{\mathrm{R}}=c<_{Q} a^{\mathrm{LR}}$. If on the other hand $a$ is the right child of its parent $c$ then one gets $a^{\mathrm{L}}=c$ and $a^{\mathrm{R}}=c^{\mathrm{R}}$. Thus by assertion (iv) one obtains $a<_{Q} a^{\mathrm{R}}=c^{\mathrm{R}}=a^{\mathrm{LR}}$.

(viii) The proof goes as for assertion (vii).

(ix) Relation $b<_{Q} a^{\mathrm{R}}$ and the converse of assertion (vi) imply that $b \leqslant_{Q} a$. Similarly, $a^{\mathrm{L}}<_{Q} b$ and the converse of assertion (v) yield that $a \leqslant_{Q} b$. By assertion (iii) one gets $a=b$.

$(\mathrm{x})$ Set $c_{1}:=a_{\mathrm{R}}$ and $c_{2}:=b_{\mathrm{L}}$. Then $a<_{Q} c_{1}$ and $c_{2}<_{Q} b$ by assertion (iv). If $c_{1}=b$ then $a=b^{\mathrm{L}}=c_{2}{ }^{\mathrm{L}}<_{Q} c_{2}<_{Q} c_{2}{ }^{\mathrm{R}}=b$ again by assertion (iv). In this case one can take $c=c_{2}$. Similarly if $a=c_{2}$ then $a=c_{1}{ }^{\mathrm{L}}<_{Q} c_{1}<_{Q} c_{1}{ }^{\mathrm{R}}=a^{\mathrm{R}}=b$ and one can take $c=c_{1}$. Suppose now that $c_{1} \neq b$ and $c_{2} \neq a$. If $\left|\sigma_{T}\right|(a) \leqslant\left|\sigma_{T}\right|(b)$ then using $a<_{Q} c_{2}{ }^{\mathrm{R}}$ the converse of (vi) implies that $a \leqslant \leqslant_{Q} c_{2}$. Since $\left|\sigma_{T}\right|\left(c_{2}\right)>\left|\sigma_{T}\right|(a)$ one obtains $a<_{Q} \quad c_{2}$ and hence one can set $c:=c_{2}$. Similarly one gets $c:=c_{1}<_{Q} b$ if $\left|\sigma_{T}\right|(b) \leqslant\left|\sigma_{T}\right|(a)$.

Suppose now that $V$ is a Yetter-Drinfel'd module of diagonal type with $d:=\operatorname{dim}_{k} V=2$. Let $\left\{x_{1}, x_{2}\right\}$ denote a canonical basis of $V$. Then for a full binary tree $T$ the following assignment defines a unique map $\tau_{0}: \bar{N}(T) \rightarrow V^{\otimes}$ :

(i) $\tau_{0}\left({ }^{\prime} L '\right):=x_{2}, \tau_{0}\left({ }^{\prime} R '\right):=x_{1}$;

(ii) If $a \in N(T)$ then $\tau_{0}(a):=\tau_{0}\left(a^{\mathrm{R}}\right) \tau_{0}\left(a^{\mathrm{L}}\right)-\chi\left(\tau_{0}\left(a^{\mathrm{R}}\right), \tau_{0}\left(a^{\mathrm{L}}\right)\right) \tau_{0}\left(a^{\mathrm{L}}\right) \tau_{0}\left(a^{\mathrm{R}}\right)$. 


\section{RANK 2 NiChOlS ALGEBRAS OF DiAGONAL TYPE}

Note that if $a \in \bar{N}(T)$ and $\sigma_{T}(a)=(r, s)$ then $\operatorname{deg} \tau_{0}(a)=r \mathbf{e}_{1}+s \mathbf{e}_{2}$. To shorten notation we will write $\chi(a, b)$ and $g(a)$ instead of $\chi\left(\tau_{0}(a), \tau_{0}(b)\right)$ and $g\left(\tau_{0}(a)\right)$, respectively, for any $a, b \in \bar{N}(T)$. Let $\tau: \bar{N}(T) \rightarrow \mathcal{B}(V)$ denote the composition of $\tau_{0}$ with the canonical map $V^{\otimes} \rightarrow \mathcal{B}(V)$.

Example 6.1. Again consider the full binary tree in Figure 1. One obtains the following expressions in $\mathcal{B}(V)$ :

$$
\begin{gathered}
\tau\left(a^{\mathrm{L}}\right)=x_{1} x_{2}-q_{12} x_{2} x_{1}, \\
\tau\left(d^{\mathrm{L}}\right)=x_{1} \tau\left(a^{\mathrm{L}}\right)-q_{11} q_{12} \tau\left(a^{\mathrm{L}}\right) x_{1}, \\
\tau\left(a^{\mathrm{R}}\right)=\tau\left(d^{\mathrm{L}}\right) \tau\left(a^{\mathrm{L}}\right)-q_{11}^{2} q_{12}^{2} q_{21} q_{22} \tau\left(a^{\mathrm{L}}\right) \tau\left(d^{\mathrm{L}}\right) .
\end{gathered}
$$

Definition 6.2. Let $n \in \mathbb{N}_{0}, T$ a full binary tree, $V$ a Yetter-Drinfel'd module of diagonal type with $\operatorname{dim}_{k} V=2$ and with canonical basis $\left\{x_{1}, x_{2}\right\}$, and let $\mathcal{B}(V)$ denote the corresponding Nichols algebra. We say that $\mathcal{B}(V)$ is of type $T$ in degree $n$ if the sets

$$
\left\{\prod_{a \in \bar{N}_{2}(T)} \tau(a)^{i_{a}} \mid 0 \leqslant i_{a}<\text { ord } \chi(a, a) \forall a \in \bar{N}_{2}(T), \sum_{a \in \bar{N}_{2}(T)} i_{a}\left|\sigma_{T}\right|(a) \leqslant m\right\}
$$

form a basis of the vector spaces $\bigoplus_{i=0}^{m} \mathcal{B}(V)_{i}$, respectively, for all $m \leqslant n$. Here the elements of $\bar{N}_{2}(T)$ are ordered with respect to the order $<_{Q}$, and ord $\chi(a, a)$ is the order of $\chi(a, a)$ in the multiplicative group $k^{*}$ if $\chi(a, a) \neq 1$ is a root of 1 , and $\infty$ otherwise. Further, we say that $\mathcal{B}(V)$ is of type $T$ if $\mathcal{B}(V)$ is of type $T$ in degree $n$ for all $n \in \mathbb{N}$.

Note that $\mathcal{B}(V)$ is of type $T$ in degree 0 for any full binary tree $T$. Further, if $\mathcal{B}(V)$ is of type $T$ for a full binary tree $T$ and ord $\chi(a, a)<\infty$ for all $a \in \bar{N}_{2}(T)$ then $\mathcal{B}(V)$ is finite dimensional. More exactly, one gets $\operatorname{dim}_{k} \mathcal{B}(V)=\prod_{a \in \bar{N}_{2}(T)}$ ord $\chi(a, a)$.

Lemma 6.2. Let $T$ be a full binary tree.

(i) There exists a unique map $\gamma: \bar{N}(T) \rightarrow \mathbb{X}$ such that $\gamma\left({ }^{\prime} L '\right)=\alpha_{1}, \gamma\left({ }^{\prime} R\right.$ ') $=\alpha_{2}$, and $\gamma(a)=$ $\gamma\left(a^{\mathrm{L}}\right) \gamma\left(a^{\mathrm{R}}\right)$ for all $a \in N(T)$.

(ii) For $a \in \bar{N}(T)$ the equation $|\gamma(a)|=\left|\sigma_{T}\right|(a)$ holds.

(iii) Any word $\gamma(a), a \in \bar{N}(T)$, is a Lyndon word and $\gamma\left(a^{\mathrm{L}}\right) \gamma\left(a^{\mathrm{R}}\right)$ is the Shirshow decomposition of $\gamma(a)$ for $a \in N(T)$.

(iv) For any $a, b \in \bar{N}(T)$ the relation $\gamma(a)<\gamma(b)$ is equivalent to $a<_{Q} b$.

Proof. (i) Existence and uniqueness of $\gamma$ follow from the facts that $(\cdot)^{\mathrm{L}}$ and $(\cdot)^{\mathrm{R}}$ are well-defined maps from $N(T)$ to $\bar{N}(T)$ and $\left|\sigma_{T}\right|\left(a^{\mathrm{L}}\right)<\left|\sigma_{T}\right|(a),\left|\sigma_{T}\right|\left(a^{\mathrm{R}}\right)<\left|\sigma_{T}\right|(a)$ for all $a \in N(T)$.

(ii) This follows immediately from the definition of $\gamma$ and $\left|\sigma_{T}\right|$.

(iii), (iv) We use induction on $\left|\sigma_{T}\right|(a)$ and $\max \left\{\left|\sigma_{T}\right|(a),\left|\sigma_{T}\right|(b)\right\}$, respectively. If $a=$ ' $L$ ' or $a=$ ' $R$ ' then $\gamma(a)$ is a Lyndon word. Further, if $\left|\sigma_{T}\right|(a)=\left|\sigma_{T}\right|(b)=1$ then $a, b \in\left\{{ }^{\prime} L\right.$ ', ' $R$ ' $\}$ and hence $a<_{Q} b$ is equivalent to $a={ }^{\prime} L$ ', $b=$ ' $R$ ' which holds if and only if $\gamma(a)=\alpha_{1}<\alpha_{2}=\gamma(b)$.

Assume now that assertions (iii) and (iv) hold whenever $a, b \in \bar{N}(T),\left|\sigma_{T}\right|(a) \leqslant n$, and $\left|\sigma_{T}\right|(b) \leqslant$ $n$ for some $n \in \mathbb{N}$. If $a \in N(T)$ then by induction hypothesis $\gamma\left(a^{\mathrm{L}}\right)$ and $\gamma\left(a^{\mathrm{R}}\right)$ are Lyndon words. Since $a^{\mathrm{L}}<_{Q} a^{\mathrm{R}}$ we also have $\gamma\left(a^{\mathrm{L}}\right)<\gamma\left(a^{\mathrm{R}}\right)$. Thus $\gamma\left(a^{\mathrm{L}}\right) \gamma\left(a^{\mathrm{R}}\right)$ is a Lyndon word by Proposition 5.1(ii). This proves the induction step of the first part of assertion (iii).

Now we prove assertion (iv) in the case $\left|\sigma_{T}\right|(a)=n+1,\left|\sigma_{T}\right|(b) \leqslant n$. The proof for $\left|\sigma_{T}\right|(b)=n+1$ is completely analogous. Let $\left(a_{1}, a_{2}, \ldots, a_{m}\right)$ denote the set of nodes of $T$ with $\left|\sigma_{T}\right|\left(a_{i}\right) \leqslant n$ in increasing order with respect to $<_{Q}$. By Lemma 6.1(ix) the node $a \in N(T)$ is the unique $c \in \bar{N}(T)$ such that $\left|\sigma_{T}\right|(c) \leqslant n+1$ and $a^{\mathrm{L}}<_{Q} c<_{Q} a^{\mathrm{R}}$. Thus there exists $i \in \mathbb{N}$ such that $a^{\mathrm{L}}=a_{i}$ and $a^{\mathrm{R}}=a_{i+1}$. On the other hand, the induction hypothesis gives that $\gamma\left(a_{j}\right)<\gamma\left(a_{l}\right)$ if and only if $j<l$. Now note that $\gamma\left(a_{i}\right)<\gamma(a)=\gamma\left(a_{i}\right) \gamma\left(a_{i+1}\right)<\gamma\left(a_{i+1}\right)$ as $\gamma(a)$ is a Lyndon word. 


\section{HECKENBERGER}

It remains to show that $\gamma\left(a^{\mathrm{L}}\right) \gamma\left(a^{\mathrm{R}}\right)$ is the Shirshow decomposition of $\gamma(a)$ where $\left|\sigma_{T}\right|(a)=n+1$. If $a^{\mathrm{L}}=$ ' $L$ ' then $\left|\gamma\left(a^{\mathrm{L}}\right)\right|=1$ and we are done. Otherwise $a^{\mathrm{L}} \in N(T)$ and Lemma 6.1(vi) and (vii) give $a^{\mathrm{R}} \leqslant{ }_{Q} a^{\mathrm{LR}}$. Therefore $\gamma\left(a^{\mathrm{R}}\right) \leqslant \gamma\left(a^{\mathrm{LR}}\right)$ by the induction hypothesis for assertion (iv). Further, the induction hypothesis of assertion (iii) tells us that $\gamma\left(a^{\mathrm{LL}}\right) \gamma\left(a^{\mathrm{LR}}\right)$ is the Shirshow decomposition of $\gamma\left(a^{\mathrm{L}}\right)$. Thus Proposition 5.2 for $u=\gamma(a)$ together with the last two relations give the claim.

Our aim in this section is to give a computable criterion which ensures that the Nichols algebras in Theorem 7.1 are of the given type. To do so we have to introduce additional notation which will be needed only for $\mathcal{A}=\mathcal{B}(V)$. Recall the definitions above Theorem 5.1.

For a Lyndon word $u$ and $n \in \mathbb{N}$ let $\mathcal{F}(u)_{n}$ denote the $k G$-module

$$
\mathcal{F}(u)_{n}:=\left(S(u) \cap\left(\mathcal{B}(V) S(u)_{<}^{+}+\mathcal{B}(V)[u]^{2}\right) \cap \bigoplus_{m=1}^{n} \mathcal{B}(V)_{m}\right) \# k G
$$

and set $\mathcal{F}(u)_{0}=\{0\}$. By Corollary 5.1 one obtains that

$$
\mathcal{F}(u)_{n}=\left(\left(S(u) S(u)_{<}^{+}+S(u)[u]^{2}\right) \cap \bigoplus_{m=1}^{n} \mathcal{B}(V)_{m}\right) \# k G .
$$

Let $n \in \mathbb{N}$. Suppose that there exists a full binary tree $T$ such that for any Lyndon word $u$ with $|u| \leqslant n$ the relation $h_{u}>1$ is equivalent to $u=\gamma(a)$ for some $a \in \bar{N}_{2}(T)$. The definition of $\tau$ and [.] and Lemma 6.2(iii) imply that for any $a \in \bar{N}_{2}(T)$ one has $\tau(a)=[\gamma(a)]$. Then by Corollary 5.1 with $u=\alpha_{1}$ and by Lemma 6.2(iv) the set

$$
\left\{\prod_{a \in \bar{N}_{2}(T)} \tau(a)^{i_{a}} \mid i_{a}<h_{\gamma(a)}, \sum_{a \in \bar{N}_{2}(T)} i_{a} \operatorname{totdeg}(\tau(a)) \leqslant n\right\}
$$

where the product is taken with respect to the order $<_{Q}$ of $\bar{N}_{2}(T)$ forms a basis of $\bigoplus_{m=0}^{n} \mathcal{B}(V)_{m}$.

For $a \in \bar{N}(T)$ define $p_{a}:=\chi(a, a)^{-1}$ and

$$
\lambda(a):= \begin{cases}0 & \text { if } a \notin N(T), \\ \chi\left({ }^{\prime} L,{ }^{\prime} R^{\prime}\right)^{-1}-\chi\left({ }^{\prime} R,{ }^{\prime} L '\right) & \text { if } a=\mathbf{r}, \\ \chi\left(a^{\mathrm{L}}, a^{\mathrm{R}}\right)^{-1}-\chi\left(a^{\mathrm{R}}, a^{\mathrm{L}}\right)+\lambda(b) & \text { otherwise, }\end{cases}
$$

where $b$ is the parent of $a$. Further, for any $b \in N(T)$ with $b^{\mathrm{L}} \in N(T)$ set

$$
\mu(b):= \begin{cases}\lambda(b) & \text { if } b=b^{\mathrm{L}}{ }_{\mathrm{R}}, \\ \lambda(b) \mu\left(b^{\mathrm{R}}\right) & \text { otherwise. }\end{cases}
$$

Finally, for any $b \in N(T)$ with $c:=b^{\mathrm{L}} \in N(T)$ and $\ell^{\mathrm{R}}(b) \leqslant 2$ set

$$
\nu(b):= \begin{cases}\chi\left(c^{\mathrm{L}}, b\right)^{-1}-\chi\left(b, c^{\mathrm{L}}\right)+\lambda(b) \lambda(c)\left((2)_{p_{f}}^{-1}-(2)_{p_{c}}^{-1}\right) & \text { if } \ell^{\mathrm{R}}(b)=1, \\ \chi\left(c^{\mathrm{L}}, c_{\mathrm{R}}\right)^{-1}+\lambda(c) \lambda\left(c_{\mathrm{R}}\right)(2)_{p_{c}}^{-1}\left((2)_{p_{f}}^{-1}-(3)_{p_{c}}^{-1}\right) & \text { if } \ell^{\mathrm{R}}(b)=2,\end{cases}
$$

where $f=c^{\mathrm{R}}$, whenever all denominators are nonzero.

\section{Explicit examples}

The following theorem is the main result of this paper.

TheOREM 7.1. Let $k$ be a field of characteristic zero and $V$ a braided vector space of diagonal type with $\operatorname{dim}_{k} V=2$. Suppose that the generalized Dynkin diagram of $V$ appears in Table 1 and is marked with the tree Tn, where $n \in\{1,2, \ldots, 22\}$ and the full binary tree Tn is given in Appendix A. 
RANK 2 Nichols AlgEBRAS OF DiAgONAL TYPE

TABLE 1. Generalized Dynkin diagrams of rank 2 Nichols algebras.

\begin{tabular}{|c|c|c|c|}
\hline & Generalized Dynkin diagrams & Fixed parameters & Tree \\
\hline 1 & $\begin{array}{ll}q & r \\
\bigcirc & \bigcirc\end{array}$ & $q, r \in k^{*}$ & $\mathrm{~T} 1$ \\
\hline 2 & $\stackrel{q}{q} \mathrm{q}^{-1} \quad \mathrm{P}^{q}$ & $q \in k^{*} \backslash\{1\}$ & $\mathrm{T} 2$ \\
\hline 3 & $\begin{array}{cccccc}q & q^{-1} & -1 & -1 & q & -1 \\
\bigcirc & \bigcirc & \bigcirc & & \bigcirc\end{array}$ & $q \in k^{*} \backslash\{-1,1\}$ & $\mathrm{T} 2 \mathrm{~T} 2$ \\
\hline 4 & $\stackrel{q}{\bigcirc^{-2}} \stackrel{q}{-2}^{q^{2}} \bigcirc$ & $q \in k^{*} \backslash\{-1,1\}$ & T3 \\
\hline 5 & 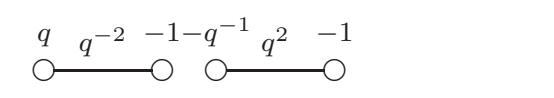 & $\begin{array}{l}q \in k^{*} \backslash\{-1,1\} \\
q \notin R_{4}\end{array}$ & T3 T3 \\
\hline 6 & 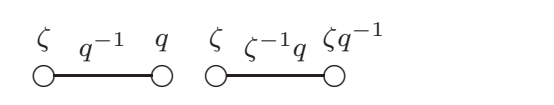 & $\begin{array}{l}\zeta \in R_{3} \\
q \in k^{*} \backslash\left\{1, \zeta, \zeta^{2}\right\}\end{array}$ & T3 T3 \\
\hline 7 & $\overbrace{}^{\zeta}-\zeta^{-1} \zeta^{-1}-\zeta^{-1-1}-\bigcirc^{\circ}$ & $\zeta \in R_{3}$ & T3 T3 \\
\hline 8 & 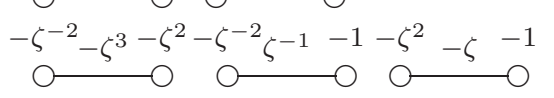 & $\zeta \in R_{12}$ & $\mathrm{~T} 4 \mathrm{~T} 5 \mathrm{~T} 5$ \\
\hline & 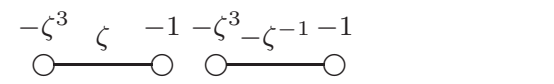 & & $\mathrm{T} 7 \mathrm{~T} 7$ \\
\hline 9 & \begin{tabular}{ccccc}
$-\zeta^{2}$ & $\zeta$ & $-\zeta^{2}-\zeta^{2}$ & $\zeta^{3}$ & $-1-\zeta^{-1}-\zeta^{3}-1$ \\
\hdashline & $\longrightarrow$
\end{tabular} & $\zeta \in R_{12}$ & T4 T5 T7 \\
\hline 10 & 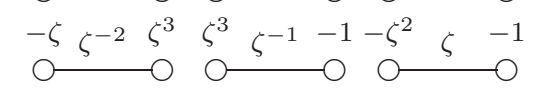 & $\zeta \in R_{9}$ & T6 T9 T14 \\
\hline 11 & $\stackrel{q}{\bigcirc^{-3} \quad q^{3}} \mathrm{P}^{\circ}$ & $\begin{array}{l}q \in k^{*} \backslash\{-1,1\} \\
q \notin R_{3}\end{array}$ & $\mathrm{~T} 8$ \\
\hline & 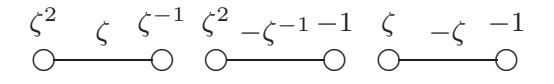 & $\zeta \in R_{8}$ & T8 T8 T8 \\
\hline 13 & $\stackrel{\zeta^{6}-\zeta^{-1-\zeta^{-4}} \zeta^{6} \quad \zeta \quad \zeta^{-1}}{\circ} \stackrel{\longrightarrow}{\circ}$ & $\zeta \in R_{24}$ & $\mathrm{~T} 10 \mathrm{~T} 13$ \\
\hline & \begin{tabular}{ccccc}
$-\zeta^{-4} \zeta^{5}$ & -1 & $\zeta$ & $\zeta^{-5}$ & -1 \\
\hdashline & ${ }^{\circ}$ & ${ }^{\circ}$
\end{tabular} & & $\mathrm{T} 17 \mathrm{~T} 21$ \\
\hline 14 & 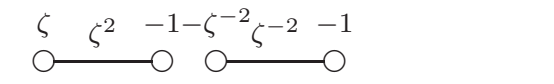 & $\zeta \in R_{5}$ & T11 T16 \\
\hline 15 & 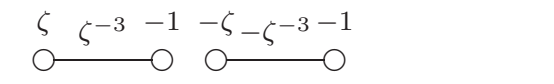 & $\zeta \in R_{20}$ & $\mathrm{~T} 11 \mathrm{~T} 11$ \\
\hline & $\stackrel{-\zeta^{-2} \zeta^{3} \quad-1-\zeta^{-2}-\zeta^{3}-1}{\circ} \bigcirc$ & & $\mathrm{T} 16 \mathrm{~T} 16$ \\
\hline 16 & $\stackrel{-\zeta-\zeta^{-3} \zeta^{5}}{\longrightarrow} \zeta^{3}-\zeta^{4-\zeta^{-4}}$ & $\zeta \in R_{15}$ & $\mathrm{~T} 12 \mathrm{~T} 15$ \\
\hline & 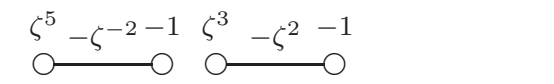 & & T18 T20 \\
\hline 17 & 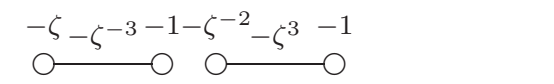 & $\zeta \in R_{7}$ & T19 T22 \\
\hline
\end{tabular}




\section{HECKENBERGER}

Then $\mathcal{B}(V)$ is of type Tn. Moreover, the algebra $\mathcal{B}(V)$ is the quotient of the tensor algebra $V^{\otimes}$ by the ideal generated by the set

$$
\begin{aligned}
\left\{\tau_{0}(a)\right. & \left.\mid a \in N_{0}(T)\right\} \cup\left\{\tau_{0}(a)^{\operatorname{ord} p_{a}} \mid a \in \bar{N}_{2}(T), 2 \leqslant \text { ord } p_{a}<\infty\right\} \\
& \cup\left\{\tau_{0}(b) \tau_{0}\left(c^{\mathrm{L}}\right)-\chi\left(b, c^{\mathrm{L}}\right) \tau_{0}\left(c^{\mathrm{L}}\right) \tau_{0}(b)\right. \\
& \left.-\mu(b) /\left(\ell^{\mathrm{R}}(b)+1\right)_{p_{c}}^{!} \tau_{0}(c)^{\ell^{\mathrm{R}}(b)+1} \mid b \in N_{2}(T), c:=b^{\mathrm{L}} \in N_{2}(T)\right\} .
\end{aligned}
$$

In particular, if $q_{11}, q_{22}$, and $q_{12} q_{21}$ are roots of 1 (and $q_{11}, q_{22}$ are different from 1 for type T1) then the Nichols algebra $\mathcal{B}(V)$ is finite dimensional.

The proof of Theorem 7.1 will be given in $\S 8$.

Remark 7.1. (1) Many of the rank 2 Nichols algebras in Theorem 7.1 have appeared in the literature previously. Those with $q_{12} q_{21}=q_{11}^{-n}, q_{22}=q_{11}^{n}, n \in\{0,1,2,3\}$, are called of finite Cartan type in [AS02]. They have type T1, T2, T3, and T8. Other examples (cf. $\S 3.3$ in [AS02]) cover essentially all Nichols algebras of type T2 and T3. Further, there exist recent computations on Nichols algebras by M. Graña and Ch. Heaton (Graña, private communication) which give a PBW basis for the examples of type T5 and T9.

(2) Theorem 7.1 does not claim that all elements in the set given in (9) are necessary to generate the defining ideal of $\mathcal{B}(V)$. For example it is known that if $(V, \sigma)$ is of Cartan type and the structure constants $q_{i i}$ are not roots of unity, then the two quantum Serre relations generate the ideal (9). On the other hand the tree T3, which is associated to the generalized Dynkin diagram in row 4 of Table 1, contains three nodes in $N_{0}(T)$.

One should take into account that many of the mentioned generators of the defining ideal of $\mathcal{B}(V)$ may become redundant by various different reasons. This is also why more explicit formulas for relations will be omitted. However in general none of the three types of elements in (9) can be avoided completely. The quantum Serre relations are clearly necessary, and root vector relations are known to be needed for Nichols algebras of Cartan type when $q_{11}$ is a root of 1 . Finally, one can check that starting with the second diagram in row 17 of Table 1, the element in the second line of (9) for $b=\mathbf{r}_{\mathrm{RR}}$, which has total degree 6 , is not in the ideal generated by the other elements of (9) of degree at most 6 .

The presentation given here has the advantage that it can be described with the help of a simple algorithm which works for all examples. Thus the description of a minimal set of relations, which could be relevant for example for the classification of pointed Hopf algebras via the lifting method of Andruskiewitsch and Schneider, remains an open problem.

\section{The finiteness results}

From now on let $T$ be a full binary tree such that

$$
\min \left\{\ell_{\mathrm{R}}\left(b_{\mathrm{L}}\right), \ell_{\mathrm{L}}\left(b_{\mathrm{R}}\right)\right\} \leqslant 3 \text { for all } b \in N_{2}(T),
$$

i.e. either $b_{\mathrm{LRRR}} \notin N(T)$ or $b_{\mathrm{RLLL}} \notin N(T)$. Note that all binary trees in Appendix A satisfy this condition.

Definition 8.1. We call a triple $(T, V, n)$, where $n \in \mathbb{N}_{0}$ and $V \in{ }_{k G}^{k G} \mathcal{Y D}$ is a two-dimensional Yetter-Drinfel'd module of diagonal type, admissible if the following conditions are satisfied.

(i) For $a \in N(T)$ with $\left|\sigma_{T}\right|(a) \leqslant n$ relation $a \in N_{2}(T)$ is equivalent to $\lambda(a) \neq 0$.

(ii) Either $N_{2}(T)$ is empty or the numbers $p_{a}$ for $a \in \bar{N}_{2}(T),\left|\sigma_{T}\right|(a) \leqslant n$, are different from 1 .

(iii) If $a \in N_{2}(T), a_{\mathrm{L}} \in N_{2}(T)$, and $\left|\sigma_{T}\right|\left(a_{\mathrm{L}}\right) \leqslant n$ then $p_{a} \neq-1, p_{a^{\mathrm{R}}} \neq-1$. 


\section{RANK 2 NiChOlS ALGEBRAS OF DiAGONAL TYPE}

(iv) For all $b \in N_{2}(T)$ with $c:=b^{\mathrm{L}} \in N_{2}(T)$ and $\left|\sigma_{T}\right|(b)+\left|\sigma_{T}\right|\left(b^{\mathrm{LL}}\right) \leqslant n$ one has $\left(\ell^{\mathrm{R}}(b)+1\right)_{p_{c}} \neq 0$ and either $\ell_{\mathrm{R}}\left(c_{\mathrm{L}}\right) \leqslant \ell^{\mathrm{R}}(b)$ or $\ell^{\mathrm{R}}(b) \leqslant 2, \nu(b)=0$.

Note that if $b \in N_{2}(T)$ and $c:=b^{\mathrm{L}} \in N_{2}(T)$ then $\ell^{\mathrm{R}}(b)<\ell_{\mathrm{L}}\left(c_{\mathrm{R}}\right)$. Thus by (10) for $c$ one has either $\ell^{\mathrm{R}}(b) \leqslant 2$ or $\ell_{\mathrm{R}}\left(c_{\mathrm{L}}\right) \leqslant \ell^{\mathrm{R}}(b)$.

Proposition 8.1. Let $T$ denote a full binary tree and $V \in{ }_{k G}^{k G} \mathcal{Y D}$ a two-dimensional YetterDrinfel'd module of diagonal type. Let $n \in \mathbb{N}_{0}$. If $(T, V, n)$ is admissible then $\mathcal{B}(V)$ is of type $T$ in degree $n$ and all homogeneous relations of $\mathcal{B}(V)$ of degree at most $n$ are elements of the ideal of $V^{\otimes}$ generated by the set

$$
\begin{aligned}
& \left\{\tau_{0}(a)\left|a \in N_{0}(T),\right| \sigma_{T} \mid(a) \leqslant n\right\} \\
& \quad \cup\left\{\tau_{0}(a)^{\operatorname{ord} p_{a}}\left|a \in \bar{N}_{2}(T),\right| \sigma_{T} \mid(a) \cdot \operatorname{ord} p_{a} \leqslant n\right\} \\
& \cup\left\{\tau_{0}(b) \tau_{0}\left(c^{\mathrm{L}}\right)-\chi\left(b, c^{\mathrm{L}}\right) \tau_{0}\left(c^{\mathrm{L}}\right) \tau_{0}(b)-\mu(b) /\left(\ell^{\mathrm{R}}(b)+1\right)_{p_{c}}^{!} \tau_{0}(c)^{\ell^{\mathrm{R}}(b)+1} \mid b \in N_{2}(T),\right. \\
& \left.\quad c:=b^{\mathrm{L}} \in N_{2}(T),\left|\sigma_{T}\right|(b)+\left|\sigma_{T}\right|\left(c^{\mathrm{L}}\right) \leqslant n\right\} .
\end{aligned}
$$

The following corollary is an immediate consequence of Proposition 8.1. It will be our main tool to prove Theorem 7.1 .

COROLlary 8.1. Let $T$ denote a full binary tree and $V \in{ }_{k G}^{k G} \mathcal{Y D}$ a two-dimensional Yetter-Drinfel'd module of diagonal type. If $(T, V, n)$ is admissible for all $n \in \mathbb{N}$ then $\mathcal{B}(V)$ is of type $T$ and all relations of $\mathcal{B}(V)$ are elements of the ideal of $V^{\otimes}$ generated by the set

$$
\begin{aligned}
& \left\{\tau_{0}(a) \mid a \in N_{0}(T)\right\} \\
& \quad \cup\left\{\tau_{0}(a)^{\operatorname{ord} p_{a}} \mid a \in \bar{N}_{2}(T), 2 \leqslant \text { ord } p_{a}<\infty\right\} \\
& \cup\left\{\tau_{0}(b) \tau_{0}\left(c^{\mathrm{L}}\right)-\chi\left(b, c^{\mathrm{L}}\right) \tau_{0}\left(c^{\mathrm{L}}\right) \tau_{0}(b)-\mu(b) /\left(\ell^{\mathrm{R}}(b)+1\right)_{p_{c}}^{!} \tau_{0}(c)^{\ell^{\mathrm{R}}(b)+1} \mid b \in N_{2}(T),\right. \\
& \left.\quad c:=b^{\mathrm{L}} \in N_{2}(T)\right\} .
\end{aligned}
$$

Proof of Proposition 8.1. We proceed by induction over $n$. As noted previously the assertion is true for $n=0$. Assume that Proposition 8.1 is valid for $(T, V, n-1)$ and that $(T, V, n)$ is admissible. By Corollary 5.1 for $\mathcal{A}=V^{\otimes}$ and with $u=\alpha_{1}$ and by Lemma 6.2 it suffices to prove that the following assertions hold.

(a) If $a \in \bar{N}_{2}(T)$ and $\left|\sigma_{T}\right|(a) \leqslant n$ then $h_{\gamma(a)} \geqslant \min \left\{\operatorname{ord} p_{a},(n+1) /\left|\sigma_{T}\right|(a)\right\}$. If $n \geqslant\left|\sigma_{T}\right|(a) \cdot \operatorname{ord} p_{a}$ then $[\gamma(a)]^{\text {ord } p_{a}}=0$.

(b) If $a \in N_{0}(T)$ and $\left|\sigma_{T}\right|(a) \leqslant n$ then $[\gamma(a)]=0$.

(c) If $u$ is a Lyndon word, $2 \leqslant|u| \leqslant n$, and $u=\gamma(a) \gamma(b)$ is the Shirshow decomposition of $u$ with $a, b \in \bar{N}_{2}(T), a=c^{\mathrm{L}}$, where $c=b^{\mathrm{L}}$, then $[u]=\mu(b) /\left(\ell^{\mathrm{R}}(b)+1\right)_{p_{c}}^{!} \tau(c)^{\ell^{\mathrm{R}}(b)+1}$ and $u<\gamma(c)$.

(d) If $u$ is a Lyndon word, $|u| \leqslant n, u \notin\{\gamma(a) \mid a \in \bar{N}(T)\}$, and $u$ is not as in assertion (c), then $h_{u}=1$ and the relation corresponding to $u$ (see the definition of $h_{u}$ below Lemma 5.3) can be obtained from those given in Proposition 8.1 for $(T, V, n-1)$.

In order to prove assertions (a)-(d) we additionally use the following induction hypotheses which will be proven after the proof of assertions (a)-(d).

(e) If $a \in N(T), m:=\left|\sigma_{T}\right|(a) \leqslant n$, and $u=\gamma(a)$ has Shirshow decomposition $u=v w$ then

$$
\Delta(\iota([u]))-\iota([u]) \otimes 1-g([u])^{-1} \otimes \iota([u])-\lambda(a) g([v])^{-1} \iota([w]) \otimes \iota([v]) \in \iota\left(\mathcal{F}(w)_{m-1}\right) \otimes \mathcal{B}\left(V^{*}\right) .
$$

(f) If $a, b \in \bar{N}(T)$ such that $\left|\sigma_{T}\right|(a) \leqslant n,\left|\sigma_{T}\right|(b) \leqslant n$, and $a<_{Q} b$ then $\langle\iota(\tau(b)), \tau(a)\rangle=0$. 


\section{HECKENBERGER}

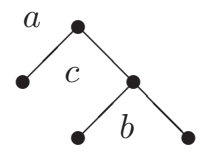

FiguRE 3. First setting in assertion (h).

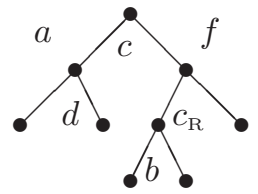

FiguRE 4. Second setting in assertion (h).

(g) If $a \in N(T)$ and $\left|\sigma_{T}\right|(a) \leqslant n$ then the following equations hold:

$$
\begin{gathered}
\left\langle\iota\left(\tau\left(a^{\mathrm{L}}\right)\right), \tau(a)\right\rangle=\lambda(a)\left\langle\iota\left(\tau\left(a^{\mathrm{L}}\right)\right), \tau\left(a^{\mathrm{L}}\right)\right\rangle \tau\left(a^{\mathrm{R}}\right), \\
\langle\iota(\tau(a)), \tau(a)\rangle=\lambda(a)\left\langle\iota\left(\tau\left(a^{\mathrm{L}}\right)\right), \tau\left(a^{\mathrm{L}}\right)\right\rangle\left\langle\iota\left(\tau\left(a^{\mathrm{R}}\right)\right), \tau\left(a^{\mathrm{R}}\right)\right\rangle .
\end{gathered}
$$

(h) Suppose that $b \in N_{2}(T), c:=b^{\mathrm{L}} \in N(T)$, and $\left|\sigma_{T}\right|(b)+\left|\sigma_{T}\right|\left(c^{\mathrm{L}}\right) \leqslant n$. Set $a:=c^{\mathrm{L}}$. If $\ell^{\mathrm{R}}(b)=1$ then $b=c_{\mathrm{R}}$ (see Figure $3^{2}$ ) and one has

$$
\left\langle\iota(\tau(a)), \tau(b) \tau(a)-\chi(b, a) \tau(a) \tau(b)-\frac{\lambda(b)}{(2)_{p_{c}}} \tau(c)^{2}\right\rangle=\langle\iota(\tau(a)), \tau(a)\rangle \nu(b) \tau(b) .
$$

If $\ell^{\mathrm{R}}(b)=2$ then $b=c_{\mathrm{RL}}$ and with $d:=c_{\mathrm{L}}$ (see Figure $4^{2}$ ) one has

$$
\left\langle\iota(\tau(d)), \tau(b) \tau(a)-\chi(b, a) \tau(a) \tau(b)-\frac{\mu(b)}{(3)_{p_{c}}^{!}} \tau(c)^{3}\right\rangle=\lambda(b)\langle\iota(\tau(d)), \tau(d)\rangle \nu(b) \tau\left(c_{\mathrm{R}}\right) .
$$

Note that for $n=0$ all assertions (a)-(h) are trivially fulfilled and hence we may start with the induction step.

To assertion (d). Suppose that $u$ is a Lyndon word with $|u|=n \geqslant 2$ and Shirshow decomposition $u=v w$ and $u$ is as in assertion (d). If $h_{v}=1$ or $h_{w}=1$ then $h_{u}=1$ by Lemma 5.2 and Proposition 5.3. More precisely, in order to ensure $h_{u}=1$ one does not need a new relation. Otherwise by induction hypothesis there exist $a, b \in \bar{N}_{2}(T)$ such that $\gamma(a)=v, \gamma(b)=w$. Note that since $\sigma_{T}(a)=\operatorname{deg}([v])$ and $\sigma_{T}(b)=\operatorname{deg}([w])$ Lemma 6.1(iii) implies that $a$ and $b$ are uniquely determined by $\gamma(a)$ and $\gamma(b)$, respectively.

Since $u$ is a Lyndon word we have also $v<w$ and hence $a<_{Q} b$ by Lemma 6.2(iv). Further, Proposition 5.2 and Lemma 6.2(iv) imply that either $a={ }^{\prime} L$ ' or $a \in N_{2}(T)$ and $b \leqslant_{Q} a^{\mathrm{R}}$. Assume for a moment that $a \in N_{2}(T)$ and $b=a^{\mathrm{R}}$ and set $c:=a_{\mathrm{R}}$. Then $a=c^{\mathrm{L}}, b=c^{\mathrm{R}}$, and $\left|\sigma_{T}\right|(c)=n$ and hence $u=\gamma(c)$. This case is covered by assertions (a) and (b). Similarly, if $a={ }^{~ '} L$ ' and $b=$ ' $R$ ' then $n=2$ and $u=\gamma(\mathbf{r})$ which again belongs to assertion (a) or (b). Therefore one has either $a=$ ' $L$ ' and $b \in N_{2}(T)$ or $a, b \in N_{2}(T)$ and $a<_{Q} b<_{Q} a^{\mathrm{R}}$.

Now we prove that $a<_{Q} b^{\mathrm{L}}$. First note that since $a<_{Q} b$, Lemma 6.1(vi) implies the relation $\left|\sigma_{T}\right|(a)<\left|\sigma_{T}\right|(b)$ in the case $a \in N_{2}(T)$. If $a=$ ' $L$ ' then this relation is trivial. Therefore one gets $a \leqslant \leqslant_{Q} b^{\mathrm{L}}$ by Lemma $6.1(\mathrm{v})$. Assume for a moment that $a=b^{\mathrm{L}}$ and set $c:=b_{\mathrm{L}}$. Then $a=c^{\mathrm{L}}$ and $b=c^{\mathrm{R}}$ and hence $u=\gamma(c)$ which is covered by assertions (a) and (b). Thus we arrive at the situation that $b \in N_{2}(T), a<_{Q} b^{\mathrm{L}}$, and either $a={ }^{\prime} L$ ' or $a \in N_{2}(T)$ and $b<_{Q} a^{\mathrm{R}}$.

\footnotetext{
${ }^{2}$ The labels in Figures 3 and 4 belong to the nodes above them.
} 


\section{RANK 2 NiChOlS ALGEBRAS OF DiAGONAL TYPE}

If $a=$ ' $L$ ' then $a<_{Q} b^{\mathrm{L}}$ implies that $\left|\sigma_{T}\right|(a)<\left|\sigma_{T}\right|\left(b^{\mathrm{L}}\right)$. If $a \in N_{2}(T)$ then the same relation follows from $a<_{Q} b^{\mathrm{L}}$ and Lemma 6.1(vi) as $b^{\mathrm{L}}<_{Q} b<_{Q} a^{\mathrm{R}}$. Therefore Lemma 6.1(v) gives $a \leqslant_{Q} b^{\mathrm{LL}}$. The case $a=b^{\mathrm{LL}}$ can be omitted as it is exactly the situation in assertion (c). Otherwise set $u_{1}=\gamma(a) \gamma\left(b^{\mathrm{L}}\right)$. Note that $u_{1}$ is a Lyndon word by Proposition 5.1(ii). We show that $h_{u_{1}}=1$ holds which proves assertion (d) by Lemma 5.2 and Proposition 5.3.

Suppose that $h_{u_{1}}>1$. As the length of $u_{1}$ is less than $n$ but at least 2 by induction hypothesis (a) there exists $c \in N_{2}(T)$ such that $u_{1}=\gamma(c)$. Since $\gamma(a)<u_{1}<\gamma\left(b^{\mathrm{L}}\right)$ one obtains $a<_{Q} c<_{Q} b^{\mathrm{L}}$. By Lemma 6.1(iv) and (v) this implies that $a \leqslant_{Q} c^{\mathrm{L}}$. As $\gamma\left(c^{\mathrm{L}}\right) \gamma\left(c^{\mathrm{R}}\right)$ is the Shirshow decomposition of $\gamma(c)$ by Lemma 6.2(iii) the equation $\gamma(a) \gamma\left(b^{\mathrm{L}}\right)=\gamma\left(c^{\mathrm{L}}\right) \gamma\left(c^{\mathrm{R}}\right)$ implies that $\left|\gamma\left(b^{\mathrm{L}}\right)\right| \leqslant\left|\gamma\left(c^{\mathrm{R}}\right)\right|$ and hence $\gamma\left(c^{\mathrm{L}}\right) \leqslant \gamma(a)$. Thus $a \leqslant Q c^{\mathrm{L}}$, Lemma 6.2(iv), and Lemma 6.1(iii) imply that $c^{\mathrm{L}}=a$ and $c^{\mathrm{R}}=b^{\mathrm{L}}$. Now we have $c^{\mathrm{L}}=a<_{Q} b^{\mathrm{LL}}<_{Q} b^{\mathrm{L}}=c^{\mathrm{R}},\left|\sigma_{T}\right|\left(b^{\mathrm{LL}}\right)<\left|\sigma_{T}\right|\left(b^{\mathrm{L}}\right)$, and $\left|\sigma_{T}\right|(c)>\left|\sigma_{T}\right|\left(b^{\mathrm{L}}\right)$. Then Lemma 6.1(ix) applied to the pair $\left(c, b^{\mathrm{LL}}\right)$ gives a contradiction.

To assertion (c). The relation $\gamma(a) \gamma(b)<\gamma(c)$ follows from $\gamma(c)=\gamma(a) \gamma\left(c^{\mathrm{R}}\right)$ and $b<_{Q} b^{\mathrm{LR}}=c^{\mathrm{R}}$ (see Lemma 6.1(vii)).

Set $U:=\tau(b) \tau(a)-\chi(b, a) \tau(a) \tau(b)-\mu(b) /\left(\ell^{\mathrm{R}}(b)+1\right)_{p_{c}}^{!} \tau(c)^{\ell^{\mathrm{R}}(b)+1}$. First note that using assertions (e), (f), and (g) one obtains

$$
\begin{aligned}
&\left\langle\iota(\tau(c)), \tau(b) \tau(a)-\chi(b, a) \tau(a) \tau(b)-\mu(b) /\left(\ell^{\mathrm{R}}(b)+1\right)_{p_{c}}^{!} \tau(c)^{\ell^{\mathrm{R}}(b)+1}\right\rangle \\
&=\langle\iota(\tau(c)), \tau(b)\rangle \tau(a)-\chi\left(b^{\mathrm{R}}, a\right) \tau(a)\langle\iota(\tau(c)), \tau(b)\rangle \\
& \quad-\left(\mu(b) /\left(\ell^{\mathrm{R}}(b)+1\right)_{p_{c}}\right)\left(\ell^{\mathrm{R}}(b)+1\right)_{p_{c}}\langle\iota(\tau(c)), \tau(c)\rangle \tau(c)^{\ell^{\mathrm{R}}(b)} \\
&=\langle\iota(\tau(c)), \tau(c)\rangle\left(\lambda(b) \tau\left(b^{\mathrm{R}}\right) \tau(a)-\lambda(b) \chi\left(b^{\mathrm{R}}, a\right) \tau(a) \tau\left(b^{\mathrm{R}}\right)-\mu(b) /\left(\ell^{\mathrm{R}}(b)\right) !\right. \\
& p_{c}\left.\tau(c)^{\ell^{\mathrm{R}}(b)}\right) .
\end{aligned}
$$

If $\ell^{\mathrm{R}}(b)=1$ then $b^{\mathrm{R}}=c^{\mathrm{R}}, \tau\left(b^{\mathrm{R}}\right) \tau(a)-\chi\left(b^{\mathrm{R}}, a\right) \tau(a) \tau\left(b^{\mathrm{R}}\right)=\tau(c)$, and $\mu(b)=\lambda(b)$. Otherwise $\mu(b)=\lambda(b) \mu\left(b^{\mathrm{R}}\right)$ and we can use induction hypothesis (c). In both cases one obtains $\langle\iota(\tau(c)), U\rangle=0$. Thus by assertion (f) one gets $\langle\iota(\tau(d)), U\rangle=0$ for all $d \in \bar{N}_{2}(T)$ with $c \leqslant \leqslant_{Q} d$. Hence it suffices to show that $\left\langle\iota\left(\left[u^{\prime}\right]\right), U\right\rangle=0$ for all Lyndon words $u^{\prime}$ with $\operatorname{deg}\left(\left[u^{\prime}\right]\right)=\operatorname{deg}(U)$ and that equation $\left\langle\iota\left(\tau\left(a_{1}\right) \tau\left(a_{2}\right) \cdots \tau\left(a_{m}\right)\right), U\right\rangle=0$ holds whenever $a_{i} \in \bar{N}_{2}(T), a_{1} \leqslant_{Q} a_{2} \leqslant_{Q} \cdots \leqslant_{Q} a_{m}<_{Q} c, m \geqslant 2$, and $\sum_{i} \sigma_{T}\left(a_{i}\right)=\left(\ell^{\mathrm{R}}(b)+1\right) \sigma_{T}(c)$. In the second case set $\sigma_{T}\left(a_{i}\right):=\left(r_{i}, s_{i}\right)$. Then $a_{i}<_{Q} c$ implies $r_{i}<Q(c) s_{i}$ and hence $\sum_{i} r_{i}<Q(c) \sum_{i} s_{i}$ which is a contradiction to $\sum_{i} \sigma_{T}\left(a_{i}\right)=\left(\ell^{\mathrm{R}}(b)+1\right) \sigma_{T}(c)$. In the first case $u^{\prime} \neq \gamma(d)$ for all $d$ since the entries of $\operatorname{deg}\left(\left[u^{\prime}\right]\right)$ are not relatively prime. Thus by assertion (d) one can reduce to the case where $u^{\prime}=v^{\prime} w^{\prime}$ is the Shirshow decomposition of $u^{\prime}, v^{\prime}=$ $\gamma\left(a^{\prime}\right), w^{\prime}=\gamma\left(b^{\prime}\right)$, and $a^{\prime}=b^{\mathrm{LL}}$. Since $\left(\ell^{\mathrm{R}}(b)+1\right) \sigma_{T}(c)=\operatorname{deg}(U)=\operatorname{deg}\left(\left[v^{\prime}\right]\left[w^{\prime}\right]\right)=\left(\ell^{\mathrm{R}}\left(b^{\prime}\right)+1\right) \sigma_{T}\left(b^{\prime \mathrm{L}}\right)$ and both the entries of $\sigma_{T}(c)$ and those of $\sigma_{T}\left(b^{\prime \mathrm{L}}\right)$ are relatively prime one gets from Lemma 6.1(iii) the equation $c=b^{\prime \mathrm{L}}$. Again by Lemma 6.1(iii) this yields $a=c^{\mathrm{L}}=b^{\prime \mathrm{LL}}=a^{\prime}$ and hence $b=b^{\prime}$ and $u^{\prime}=u$.

Note that $\langle\iota(\tau(b)), U\rangle=0$ and hence it remains to check that the relation $\langle\iota(\tau(b) \tau(a)), U\rangle=0$ holds. Using $\tau(b)=\tau\left(b^{\mathrm{R}}\right) \tau(c)-\chi\left(b^{\mathrm{R}}, c\right) \tau(c) \tau\left(b^{\mathrm{R}}\right)$ this implies that it remains to check the equation $\left\langle\iota\left(\tau\left(b^{\mathrm{R}}\right) \tau\left(c_{\mathrm{L}}\right)\right), U\right\rangle=0$. Set $c_{1}:=c_{\mathrm{L}}$ and $c_{i+1}:=c_{i \mathrm{R}}$ for $1 \leqslant i<\ell_{\mathrm{R}}\left(c_{1}\right)$. Further, set $b_{1}:=b^{\mathrm{R}}$ and $b_{i+1}:=b_{i}{ }^{\mathrm{R}}$ for $1 \leqslant i<\ell^{\mathrm{R}}(b)$. Then using assertion (c) for $(T, V, n-1)$, the definition of $\tau$, and Lemma 6.2(iii) one can show by induction over $i$ that for all $i \leqslant \min \left\{\ell^{\mathrm{R}}(b), \ell_{\mathrm{R}}\left(c_{1}\right)\right\}$ the assertions $\langle\iota(\tau(b) \tau(a)), U\rangle=0$ and $\left\langle\iota\left(\tau\left(b_{i}\right) \tau\left(c_{i}\right)\right), U\right\rangle=0$ are equivalent. Now we use that $(T, V, n)$ is admissible. More exactly, we have either $\ell_{\mathrm{R}}\left(c_{1}\right) \leqslant \ell^{\mathrm{R}}(b)$ or $\ell^{\mathrm{R}}(b) \leqslant 2$. In the first case one has $\tau\left(c_{i}\right)=0$ for $i=\ell_{\mathrm{R}}\left(c_{1}\right)$. Otherwise assertion (h) gives $\left\langle\iota\left(\tau\left(c_{i}\right)\right), U\right\rangle=0$ for $i=\ell^{\mathrm{R}}(b)-1$ where $c_{0}=a$. Therefore in both cases we get $\langle\iota(\tau(b) \tau(a)), U\rangle=0$ and hence $U=0$.

To assertion (b). By assertions (c), (d), (e), and (f) it suffices to show that the equations

$$
\left\langle\iota\left(\tau\left(a_{1}\right) \tau\left(a_{2}\right) \cdots \tau\left(a_{m}\right)\right), \tau(a)\right\rangle=0
$$




\section{HECKENBERGER}

hold for all $a_{1} \leqslant_{Q} a_{2} \leqslant_{Q} \cdots \leqslant_{Q} a_{m} \leqslant_{Q} a$ with $\sum_{i} \sigma_{T}\left(a_{i}\right)=\sigma_{T}(a)$. Set $\left(r_{i}, s_{i}\right):=\sigma_{T}\left(a_{i}\right)$. Then $a_{i} \leqslant Q$ implies $r_{i} \leqslant Q(a) s_{i}$ for all $i$ with equality if and only if $a_{i}=a$. Hence $\sum_{i} \sigma_{T}\left(a_{i}\right)=\sigma_{T}(a)$ implies $m=1$ and $a_{1}=a$. Note that $(T, V, n)$ is admissible and hence $\lambda(a)=0$. Thus the equation $\langle\iota(\tau(a)), \tau(a)\rangle=0$ follows from assertion $(\mathrm{g})$.

To assertion (a). Let $a \in \bar{N}_{2}(T)$ and set $u:=\gamma(a)$. Suppose that $[u]^{h}, h \in \mathbb{N}$, is a linear combination of elements $\left[u_{1}\right]\left[u_{2}\right] \cdots\left[u_{m}\right]$ with $u<u_{i}$ for $1 \leqslant i \leqslant m$. By Corollary 5.1 and the induction hypothesis one can assume that $u_{i}=\gamma\left(a_{i}\right)$ for some $a_{i}$ with $a<_{Q} a_{i}$. Set $\left(r_{i}, s_{i}\right):=\sigma_{T}\left(a_{i}\right)$ for all $i$ and $(r, s):=\sigma_{T}(a)$. Then Lemma 6.2 implies that $r_{i}>Q(a) s_{i}$ for all $i$ and hence $\left(\sum_{i} r_{i}\right)>\left(\sum_{i} s_{i}\right) Q(a)$. On the other hand, since $\tau\left(a_{1}\right) \tau\left(a_{2}\right) \cdots \tau\left(a_{m}\right)$ must have the same degree as $\tau(a)^{h}$ it follows that $(h r, h s)=\left(\sum_{i} r_{i}, \sum_{i} s_{i}\right)$ and hence $\tau(a)^{h}$ has to be zero. In particular, $0=\left\langle\iota(\tau(a)), \tau(a)^{h}\right\rangle=(h)_{p_{a}}\langle\iota(\tau(a)), \tau(a)\rangle \tau(a)^{h-1}$ by assertions (e) and (f). If $n=1$ then $\langle\iota(\tau(a)), \tau(a)\rangle=1$. Otherwise since $(T, V, n)$ is admissible one has $\lambda(a) \neq 0$. In this case assertion (g) gives $\langle\iota(\tau(a)), \tau(a)\rangle \neq 0$ and hence the first part of assertion (a) is proven. To show that $\tau(a)^{\text {ord } p_{a}}=0$ for $n \geqslant\left|\sigma_{T}\right|(a) \cdot$ ord $p_{a}$ by assertions (c) and (d) it suffices to check that $\left\langle\iota\left(\tau\left(a_{1}\right) \tau\left(a_{2}\right) \cdots \tau\left(a_{m}\right)\right), \tau(a)^{\text {ord } p_{a}}\right\rangle=0$ whenever $a_{1} \leqslant_{Q} a_{2} \leqslant_{Q} \cdots \leqslant_{Q} a_{m}<_{Q} a, a_{i} \in \bar{N}_{2}(T)$ for all $i$, and $\sum_{i} \sigma_{T}\left(a_{i}\right)=\sigma_{T}(a) \cdot \operatorname{ord} p_{a}$. However as argued at the beginning of the proof of assertion (a) such a choice of $a_{i}$ is not possible.

It remains to prove the induction step for assertions (e)-(h) $(n \rightarrow n+1)$ under the hypotheses (a)-(h) and admissibility of $(T, V, n+1)$.

Lemma 8.1. For $a \in N(T)$ with $a^{\mathrm{R}} \in N(T),\left|\sigma_{T}\right|(a)=n+1$ set $u:=\gamma(a)$ and let $u=v w$ be the Shirshow decomposition of $u$. Let $\rho \in \mathcal{B}(V)^{+} \cap \mathcal{F}\left(\gamma\left(a^{\mathrm{RR}}\right)\right)_{|w|-1}$ be a homogeneous element with respect to the $\mathbb{Z}^{2}$-grading. Then $g([w])^{-1} g(\rho) \iota(\rho) \iota([v])-\chi([w],[v]) \iota([v]) g([w])^{-1} g(\rho) \iota(\rho) \in \iota\left(\mathcal{F}(w)_{n}\right)$.

Proof. First note that

$$
\begin{aligned}
& g([w])^{-1} g(\rho) \iota(\rho) \iota([v])-\chi([w],[v]) \iota([v]) g([w])^{-1} g(\rho) \iota(\rho) \\
& \quad=g([w])^{-1} g(\rho) \iota(\rho[v]-\chi(\rho,[v])[v] \rho) \\
& \rho_{1} \rho_{2}[v]-\chi\left(\rho_{1} \rho_{2},[v]\right)[v] \rho_{1} \rho_{2} \\
& \quad=\rho_{1}\left(\rho_{2}[v]-\chi\left(\rho_{2},[v]\right)[v] \rho_{2}\right)+\chi\left(\rho_{2},[v]\right)\left(\rho_{1}[v]-\chi\left(\rho_{1},[v]\right)[v] \rho_{1}\right) \rho_{2}
\end{aligned}
$$

for $\mathbb{Z}^{2}$-homogeneous elements $\rho_{i} \in \mathcal{B}(V), i=1,2$. As $\rho \in S(v)_{<}$Lemma 5.3 implies that $\rho[v]-$ $\chi(\rho,[v])[v] \rho \in S(v)_{<}$. Further, $\rho[v],[v] \rho \in \mathcal{B}(V)_{m}$ where $m=\operatorname{totdeg}(\rho)+|v| \leqslant n$. By Lemma 6.1(ix) there exists no $b \in \bar{N}_{2}(T)$ such that $\left|\sigma_{T}\right|(b) \leqslant n$ and $a^{\mathrm{L}}<_{Q} b<_{Q} a^{\mathrm{R}}$. Thus $S(v)_{<} \cap \mathcal{B}(V)_{m}=$ $S(w) \cap \mathcal{B}(V)_{m}$. By $(*)$ and since $\rho \in S\left(\gamma\left(a^{\mathrm{RR}}\right)\right)^{+} \subset S(w)_{<}^{+}$it suffices to show that

$$
\rho[v]-\chi(\rho,[v])[v] \rho \in S(w) S(w)_{<}^{+}+S(w)[w]^{2}
$$

for $\rho=\tau\left(a^{\mathrm{RR}}\right)^{2}$ and for $\rho=\tau(b), a^{\mathrm{RR}}<_{Q} b$. By (6) relation (**) is obviously true if $|v| \geqslant|w|$. Otherwise $a$ is the left child of $a^{\mathrm{R}}$ and $a^{\mathrm{RL}}=a^{\mathrm{L}}$. If $\rho=\tau\left(a^{\mathrm{RR}}\right)^{2}$ then $\operatorname{totdeg}(\rho[v])=|v|+2\left|\sigma_{T}\right|\left(a^{\mathrm{RR}}\right)=$ $|w|+\left|\sigma_{T}\right|\left(a^{\mathrm{RR}}\right)>|w|$ and hence $(* *)$ holds. On the other hand $\operatorname{deg}\left([v] \tau\left(a^{\mathrm{RR}}\right)\right)=\operatorname{deg}([w])$ and Lemma 6.1(iii) imply that $\operatorname{deg}(\tau(b)) \neq \operatorname{deg}([w])-\operatorname{deg}([v])$ for $b \neq a^{\mathrm{RR}}$. Thus if $\rho=\tau(b), a^{\mathrm{RR}}<_{Q} b$ then again $(* *)$ is valid. This completes the proof of Lemma 8.1.

To assertion (e). Suppose that $\left|\sigma_{T}\right|(a)=n+1$. One has $a<_{Q} a^{\mathrm{R}}$ and if $a^{\mathrm{L}} \in N(T)$ then $a^{\mathrm{R}} \leqslant_{Q} a^{\mathrm{LR}}$ by Lemma 6.1 (vii) and (vi). Therefore the induction hypothesis (e) for $v$ and $w$ and Lemma 8.1 give

$$
\begin{aligned}
& \Delta(\iota([v]))-\iota([v]) \otimes 1-g\left(a^{\mathrm{L}}\right)^{-1} \otimes \iota([v])-\lambda\left(a^{\mathrm{L}}\right) g\left(a^{\mathrm{LL}}\right)^{-1} \iota\left(\tau\left(a^{\mathrm{LR}}\right)\right) \otimes \iota\left(\tau\left(a^{\mathrm{LL}}\right)\right) \\
& \quad \in \iota\left(\mathcal{F}(w)_{|v|-1}\right) \otimes \mathcal{B}\left(V^{*}\right),
\end{aligned}
$$




\section{RANK 2 NiChOlS ALGEBRAS OF DiAGONAL TYPE}

$$
\begin{aligned}
\Delta(\iota([u]))= & \Delta(\iota([w])) \Delta(\iota([v]))-\chi\left(a^{\mathrm{R}}, a^{\mathrm{L}}\right) \Delta(\iota([v])) \Delta(\iota([w])) \\
= & \iota([u]) \otimes 1+\left(\chi\left(a^{\mathrm{L}}, a^{\mathrm{R}}\right)^{-1}-\chi\left(a^{\mathrm{R}}, a^{\mathrm{L}}\right)\right) g\left(a^{\mathrm{L}}\right)^{-1} \iota([w]) \otimes \iota([v])+g(a)^{-1} \otimes \iota([u]) \\
& +\lambda\left(a^{\mathrm{L}}\right) g\left([w] \tau\left(a^{\mathrm{LL}}\right)\right)^{-1} \iota\left(\tau\left(a^{\mathrm{LR}}\right)\right) \otimes \iota\left([w] \tau\left(a^{\mathrm{LL}}\right)-\chi\left(a^{\mathrm{R}}, a^{\mathrm{LL}}\right) \tau\left(a^{\mathrm{LL}}\right)[w]\right) \\
& +\lambda\left(a^{\mathrm{R}}\right) g\left(a^{\mathrm{RL}}\right)^{-1} \iota\left(\tau\left(a^{\mathrm{RR}}\right)[v]-\chi\left(a^{\mathrm{RR}}, a^{\mathrm{L}}\right)[v] \tau\left(a^{\mathrm{RR}}\right)\right) \otimes \iota\left(\tau\left(a^{\mathrm{RL}}\right)\right)
\end{aligned}
$$

up to terms in $\iota\left(\mathcal{F}(w)_{n}\right) \otimes \mathcal{B}\left(V^{*}\right)$. Thus if $a=\mathbf{r}(T)$ then $\lambda\left(a^{\mathrm{L}}\right)=\lambda\left(a^{\mathrm{R}}\right)=0$ and one gets (11). If $a$ is the left child of its parent then either $a^{\mathrm{L}}={ }^{\prime} L$ ' or $a^{\mathrm{R}}<_{Q} a^{\mathrm{LR}}$ by Lemma 6.1(vi) and (vii). Further, $a^{\mathrm{RL}}=a^{\mathrm{L}}$ and $\tau\left(a^{\mathrm{RR}}\right)[v]-\chi\left(a^{\mathrm{RR}}, a^{\mathrm{L}}\right)[v] \tau\left(a^{\mathrm{RR}}\right)=[w]$ and again we are done. Finally if $a$ is the right child of its parent then either $a^{\mathrm{R}}={ }^{\prime} R$ ' and $\lambda\left(a^{\mathrm{R}}\right)=0$ or $|v|>|w|$ and $\tau\left(a^{\mathrm{RR}}\right)[v]-$ $\chi\left(a^{\mathrm{RR}}, a^{\mathrm{L}}\right)[v] \tau\left(a^{\mathrm{RR}}\right) \in \mathcal{F}(w)_{n}$ by $(6)$. Moreover $a^{\mathrm{LR}}=a^{\mathrm{R}}$ and $[w] \tau\left(a^{\mathrm{LL}}\right)-\chi\left(a^{\mathrm{R}}, a^{\mathrm{LL}}\right) \tau\left(a^{\mathrm{LL}}\right)[w]=[v]$. Thus (11) holds in this case as well.

To assertion (f). If $\left|\sigma_{T}\right|(a) \leqslant n$ and $\left|\sigma_{T}\right|(b) \leqslant n$ then we are done by induction hypothesis. If $n+1=\left|\sigma_{T}\right|(b)>\left|\sigma_{T}\right|(a)$ then $\langle\iota(\tau(b)), \tau(a)\rangle=0$ clearly holds. Suppose now that $\left|\sigma_{T}\right|(a)=n+1$. If $n=0$ then $a='$ ' $L$ ' and $b=$ ' $R$ ' and hence assertion (f) holds by definition of $\langle\cdot, \cdot\rangle$. Otherwise $a^{\mathrm{R}} \leqslant_{Q} b$ by Lemma $6.1(\mathrm{vi})$ and hence assertions (e) and (f) imply that

$$
\begin{aligned}
\langle\iota(\tau(b)), \tau(a)\rangle & =\left\langle\iota(\tau(b)), \tau\left(a^{\mathrm{R}}\right) \tau\left(a^{\mathrm{L}}\right)-\chi\left(a^{\mathrm{R}}, a^{\mathrm{L}}\right) \tau\left(a^{\mathrm{L}}\right) \tau\left(a^{\mathrm{R}}\right)\right\rangle \\
& =\left\langle\iota(\tau(b)), \tau\left(a^{\mathrm{R}}\right)\right\rangle a^{\mathrm{L}}-\chi\left(a^{\mathrm{R}}, a^{\mathrm{L}}\right) \chi\left(b, a^{\mathrm{L}}\right)^{-1} a^{\mathrm{L}}\left\langle\iota(\tau(b)), \tau\left(a^{\mathrm{R}}\right)\right\rangle .
\end{aligned}
$$

By assertion (f) the last expression vanishes if $a^{\mathrm{R}} \leqslant_{Q} b$.

To assertion (g). By assertion (f) and since $\tau(a)=\tau\left(a^{\mathrm{R}}\right) \tau\left(a^{\mathrm{L}}\right)-\chi\left(a^{\mathrm{R}}, a^{\mathrm{L}}\right) \tau\left(a^{\mathrm{L}}\right) \tau\left(a^{\mathrm{R}}\right)$ the second equation of assertion $(\mathrm{g})$ follows immediately from the first one.

If $a=\mathbf{r}(T)$ then one gets

$$
\left\langle\iota\left(\tau\left(a^{\mathrm{L}}\right)\right), \tau(a)\right\rangle=\left\langle y_{2}, x_{1} x_{2}-\chi\left(x_{1}, x_{2}\right) x_{2} x_{1}\right\rangle=\left(\chi\left(x_{2}, x_{1}\right)^{-1}-\chi\left(x_{1}, x_{2}\right)\right) x_{1}=\lambda(a) \tau\left(a^{\mathrm{R}}\right) .
$$

If $a=a^{\mathrm{L}}{ }_{\mathrm{R}}$ then $a^{\mathrm{LR}}=a^{\mathrm{R}}$ and $\left|\sigma_{T}\right|\left(a^{\mathrm{L}}\right)>\left|\sigma_{T}\right|\left(a^{\mathrm{R}}\right)$ and hence assertions (e) and (f) give

$$
\begin{aligned}
\left\langle\iota\left(\tau\left(a^{\mathrm{L}}\right)\right), \tau(a)\right\rangle= & \left\langle\iota\left(\tau\left(a^{\mathrm{L}}\right)\right), \tau\left(a^{\mathrm{R}}\right) \tau\left(a^{\mathrm{L}}\right)-\chi\left(a^{\mathrm{R}}, a^{\mathrm{L}}\right) \tau\left(a^{\mathrm{L}}\right) \tau\left(a^{\mathrm{R}}\right)\right\rangle \\
= & \chi\left(a^{\mathrm{L}}, a^{\mathrm{R}}\right)^{-1} \tau\left(a^{\mathrm{R}}\right)\left\langle\iota\left(\tau\left(a^{\mathrm{L}}\right)\right), \tau\left(a^{\mathrm{L}}\right)\right\rangle+\lambda\left(a^{\mathrm{L}}\right)\left\langle\iota\left(\tau\left(a^{\mathrm{R}}\right)\right), \tau\left(a^{\mathrm{R}}\right)\right\rangle\left\langle\iota\left(\tau\left(a^{\mathrm{LL}}\right)\right), \tau\left(a^{\mathrm{L}}\right)\right\rangle \\
& -\chi\left(a^{\mathrm{R}}, a^{\mathrm{L}}\right)\left\langle\iota\left(\tau\left(a^{\mathrm{L}}\right)\right), \tau\left(a^{\mathrm{L}}\right)\right\rangle \tau\left(a^{\mathrm{R}}\right) .
\end{aligned}
$$

Since $a^{\mathrm{L}} \in N_{2}(T)$ in the second summand of the last expression one can use assertion (g) for $a^{\mathrm{L}}$ and the equation $a^{\mathrm{LR}}=a^{\mathrm{R}}$. One gets

$$
\begin{aligned}
\left\langle\iota\left(\tau\left(a^{\mathrm{L}}\right)\right), \tau(a)\right\rangle & =\left(\lambda\left(a^{\mathrm{L}}\right)+\chi\left(a^{\mathrm{L}}, a^{\mathrm{R}}\right)^{-1}-\chi\left(a^{\mathrm{R}}, a^{\mathrm{L}}\right)\right)\left\langle\iota\left(\tau\left(a^{\mathrm{L}}\right)\right), \tau\left(a^{\mathrm{L}}\right)\right\rangle \tau\left(a^{\mathrm{R}}\right) \\
& =\lambda(a)\left\langle\iota\left(\tau\left(a^{\mathrm{L}}\right)\right), \tau\left(a^{\mathrm{L}}\right)\right\rangle \tau\left(a^{\mathrm{R}}\right) .
\end{aligned}
$$

Finally, if $a=a^{\mathrm{R}}{ }_{\mathrm{L}}$ then $a^{\mathrm{RL}}=a^{\mathrm{L}}$. Moreover Lemma 6.1(vii) implies that $a^{\mathrm{L}}=$ ' $L$ ' or $a^{\mathrm{R}}<_{Q}$ $a^{\mathrm{RLR}}=a^{\mathrm{LR}}$. Thus using assertions (e), (f), and (g) computations similar to the previous case lead to the desired assertion.

To assertion (h). We need the following lemma.

Lemma 8.2. Suppose that $(T, V, n+1)$ is admissible. Let $d \in N_{2}(T)$ such that $c:=d^{\mathrm{R}} \in N(T)$ and $d=c_{\mathrm{L}}$. Set $a:=c^{\mathrm{L}}=d^{\mathrm{L}}$ and $f:=c^{\mathrm{R}}$ (see Figure 4 ).

(i) If $\left|\sigma_{T}\right|(d)+\left|\sigma_{T}\right|(f) \leqslant n$ then in $\mathcal{B}(V)$ one has

$$
\tau(f) \tau(d)-\chi(f, d) \tau(d) \tau(f)=\frac{\lambda(d)}{(2)_{p_{c}}} \tau(c)^{2} .
$$




\section{HECKENBERGER}

(ii) If $\left|\sigma_{T}\right|(d)+\left|\sigma_{T}\right|(f)\left(=\left|\sigma_{T}\right|(a)+\left|\sigma_{T}\right|\left(c_{\mathrm{R}}\right)\right) \leqslant n+1$ then

$$
\left\langle\iota(\tau(a)), \tau\left(c_{\mathrm{R}}\right)\right\rangle=\frac{\lambda(c) \lambda\left(c_{\mathrm{R}}\right)}{(2)_{p_{f}}}\langle\iota(\tau(a)), \tau(a)\rangle \tau(f)^{2} .
$$

(iii) If $c_{\mathrm{R}} \in N_{2}(T)$ and $\left|\sigma_{T}\right|\left(c_{\mathrm{RL}}\right)+\left|\sigma_{T}\right|(a) \leqslant n+1$ then

$$
\left\langle\iota(\tau(d)), \tau\left(c_{\mathrm{RL}}\right)\right\rangle=\frac{\lambda\left(c_{\mathrm{R}}\right) \lambda\left(c_{\mathrm{RL}}\right) \lambda(c)\langle\iota(\tau(d)), \tau(d)\rangle}{(2)_{p_{c}}(2)_{p_{f}}} \tau(f)^{2} .
$$

Proof. (i) Note that $c^{\mathrm{L}}=d^{\mathrm{L}}$ by assumption. Further, we may use the induction hypothesis (c). One computes

$$
\begin{aligned}
\tau(f) \tau(d)-\chi(f, d) \tau(d) \tau(f)= & \tau(f)(\tau(c) \tau(a)-\chi(c, a) \tau(a) \tau(c))-\chi(f, d) \tau(d) \tau(f) \\
= & \left(\tau\left(c_{\mathrm{R}}\right)+\chi(f, c) \tau(c) \tau(f)\right) \tau(a)-\chi(c, a)(\tau(c) \\
& +\chi(f, a) \tau(a) \tau(f)) \tau(c)-\chi(f, d) \tau(d) \tau(f) \\
= & \chi\left(c_{\mathrm{R}}, a\right) \tau(a) \tau\left(c_{\mathrm{R}}\right)+\lambda\left(c_{\mathrm{R}}\right) /(2)_{p_{c}} \tau(c)^{2}+\chi(f, c) \tau(c)(\tau(c) \\
& +\chi(f, a) \tau(a) \tau(f))-\chi(c, a) \tau(c)^{2}-\chi\left(c_{\mathrm{R}}, a\right) \tau(a)\left(\tau\left(c_{\mathrm{R}}\right)\right. \\
& +\chi(f, c) \tau(c) \tau(f))-\chi(f, d) \tau(d) \tau(f) \\
= & \lambda\left(c_{\mathrm{R}}\right) /(2)_{p_{c}} \tau(c)^{2}+\chi(f, c) \tau(c)^{2}+\chi(f, d)(\tau(d)+\chi(c, a) \tau(a) \tau(c)) \tau(f) \\
& -\chi(c, a) \tau(c)^{2}-\chi\left(c_{\mathrm{R}}, a\right) \chi(f, c) \tau(a) \tau(c) \tau(f)-\chi(f, d) \tau(d) \tau(f) \\
= & \left(\lambda\left(c_{\mathrm{R}}\right)+\chi(f, c)+\chi(a, c)^{-1}-\chi(c, a)-\chi(c, f)^{-1}\right) /(2)_{p_{c}} \tau(c)^{2} .
\end{aligned}
$$

Then the defining recursion formulas for $\lambda\left(c_{\mathrm{R}}\right)$ and $\lambda(d)$ give part (i).

(ii) Note that $(2)_{p_{f}} \neq 0$ by admissibility of $(T, V, n+1)$. Further, if $\tau\left(c_{\mathrm{R}}\right)=0$ then $\lambda\left(c_{\mathrm{R}}\right)=0$ by assertion (a). Thus in this case we are done. Assume now that $c=\mathbf{r}$ or $a_{\mathrm{R}}=c$ (i.e. $a^{\mathrm{R}}=f$ ). Then one has $\langle\iota(\tau(a)), \tau(f)\rangle=0$. Using assertions (e) and (f) one gets

$$
\begin{aligned}
\left\langle\iota(\tau(a)), \tau\left(c_{\mathrm{R}}\right)\right\rangle= & \langle\iota(\tau(a)), \tau(f) \tau(c)-\chi(f, c) \tau(c) \tau(f)\rangle \\
= & \chi(a, f)^{-1} \tau(f)\langle\iota(\tau(a)), \tau(c)\rangle+\lambda(a)\langle\iota(\tau(f)), \tau(f)\rangle\left\langle\iota\left(\tau\left(a^{\mathrm{L}}\right)\right), \tau(c)\right\rangle \\
& -\chi(f, c)\langle\iota(\tau(a)), \tau(c)\rangle \tau(f) .
\end{aligned}
$$

If one starts with $a_{\mathrm{L}}$ instead of $d$ in Lemma 8.2 then part (ii) gives a formula for the second summand in the last expression. Further, assertion (g) can be used to compute $\langle\iota(\tau(a)), \tau(c)\rangle$ and $\langle\iota(\tau(a)), \tau(a)\rangle$. One obtains

$$
\begin{aligned}
\left\langle\iota(\tau(a)), \tau\left(c_{\mathrm{R}}\right)\right\rangle= & \lambda(c)\langle\iota(\tau(a)), \tau(a)\rangle\left(\chi(a, f)^{-1}-\chi(f, c)\right) \tau(f)^{2} \\
& +\lambda(a)\langle\iota(\tau(f)), \tau(f)\rangle \frac{\lambda(c) \lambda(a)}{(2)_{p_{f}}}\left\langle\iota\left(\tau\left(a^{\mathrm{L}}\right)\right), \tau\left(a^{\mathrm{L}}\right)\right\rangle \tau(f)^{2} \\
= & \frac{\lambda(c)\langle\iota(\tau(a)), \tau(a)\rangle}{(2)_{p_{f}}}\left(\chi(a, f)^{-1}+\chi(c, f)^{-1}-\chi(f, c)-\chi(f, a)+\lambda(a)\right) \tau(f)^{2} .
\end{aligned}
$$

Note that these computations make sense also in the case when $a=$ ' $L$ '. Thus the recursion formulas for $\lambda(c)$ and $\lambda\left(c_{\mathrm{R}}\right)$ give part (ii) in this case. The proof of part (ii) in the remaining case (when $f_{\mathrm{L}}=c$, i.e. $\left.c^{\mathrm{L}}=f^{\mathrm{L}},\right)$ is obtained similarly.

(iii) The proof of this part is by far the most complicated. We give only a sketch of it. Set $b:=c_{\mathrm{RL}}$. 


\section{RANK 2 NiChOlS ALGEBRAS OF DiAGONAL TYPE}

Using assertions (e), (f), (g), and Lemma 8.2(ii) one obtains

$$
\begin{aligned}
\langle\iota(\tau(a)), \tau(b)\rangle= & \frac{\lambda(c)\langle\iota(\tau(a)), \tau(a)\rangle}{(2)_{p_{f}}}\left[\left(\lambda\left(c_{\mathrm{R}}\right)-\chi\left(c_{\mathrm{R}}, c\right)(2)_{p_{f}}\right) \tau(f) \tau\left(c_{\mathrm{R}}\right)\right. \\
& \left.+\left(\chi(f, c) \lambda\left(c_{\mathrm{R}}\right)+\chi\left(a, c_{\mathrm{R}}\right)^{-1}(2)_{p_{f}}\right) \tau\left(c_{\mathrm{R}}\right) \tau(f)\right], \\
\left\langle\iota(\tau(c)), \tau(f) \tau\left(c_{\mathrm{R}}\right)\right\rangle= & \frac{\lambda\left(c_{\mathrm{R}}\right)\langle\iota(\tau(c)), \tau(c)\rangle}{(2)_{p_{f}}}\left(\lambda(c)+\chi(c, f)^{-1}(2)_{p_{f}}\right) \tau(f)^{2}, \\
\left\langle\iota(\tau(c)), \tau\left(c_{\mathrm{R}}\right) \tau(f)\right\rangle= & \lambda\left(c_{\mathrm{R}}\right)\langle\iota(\tau(c)), \tau(c)\rangle \tau(f)^{2}, \\
\langle\iota(\tau(a) \tau(c)), \tau(b)\rangle= & \frac{\lambda\left(c_{\mathrm{R}}\right) \lambda(b) \lambda(c)}{(2)_{p_{f}}}\langle\iota(\tau(c)), \tau(c)\rangle\langle\iota(\tau(a)), \tau(a)\rangle \tau(f)^{2} .
\end{aligned}
$$

By assertions (c) and (h) one obtains $\nu\left(c_{\mathrm{R}}\right)=0$. Now insert the second, third, and fourth equation into the first one and replace the summand $\lambda\left(c_{\mathrm{R}}\right) \lambda(c)(2)_{p_{f}}^{-1}$ of the product

$$
\left(\lambda\left(c_{\mathrm{R}}\right)-\chi\left(c_{\mathrm{R}}, c\right)(2)_{p_{f}}\right)\left(\lambda(c)+\chi(c, f)^{-1}(2)_{p_{f}}\right)
$$

by the expression $\lambda\left(c_{\mathrm{R}}\right) \lambda(c)(2)_{p_{c}}^{-1}-\chi\left(a, c_{\mathrm{R}}\right)^{-1}+\chi\left(c_{\mathrm{R}}, a\right)$. Then use the recursion formulas for $\lambda(d)$, $\lambda(b)$, and $\lambda\left(c_{\mathrm{R}}\right)$ to obtain part (iii). This completes the proof of Lemma 8.2.

To show the first equation of assertion (h) one can use assertions (e) and (f) to obtain

$$
\begin{aligned}
& \left\langle\iota(\tau(a)), \tau(b) \tau(a)-\chi(b, a) \tau(a) \tau(b)-\frac{\lambda(b)}{(2)_{p_{c}}} \tau(c)^{2}\right\rangle \\
& =\langle\iota(\tau(a)), \tau(b)\rangle \tau(a)+\chi(a, b)^{-1} \tau(b)\langle\iota(\tau(a)), \tau(a)\rangle \\
& \quad-\chi(b, a)\langle\iota(\tau(a)), \tau(a)\rangle \tau(b)-\chi(f, a)^{2} \tau(a)\langle\iota(\tau(a)), \tau(b)\rangle \\
& \quad-\frac{\lambda(b)}{(2)_{p_{c}}}\left(\langle\iota(\tau(a)), \tau(c)\rangle \tau(c)+\chi(a, c)^{-1} \tau(c)\langle\iota(\tau(a)), \tau(c)\rangle\right) .
\end{aligned}
$$

After inserting the formula in Lemma 8.2(ii) the latter expression becomes the sum of the expression $\langle\iota(\tau(a)), \tau(a)\rangle \nu(b) \tau(b)$ and a multiple of $\tau(c) \tau(f)$. Apply $\langle\iota(\tau(f)), \cdot\rangle$ to this equation. Since one has $\langle\iota(\tau(f)), \tau(b)\rangle=0$ and $\langle\iota(\tau(f)), \tau(c) \tau(f)\rangle=\chi(f, c)^{-1} \tau(c)\langle\iota(\tau(f)), \tau(f)\rangle$, Lemma 8.2(i) gives that the coefficient of $\tau(c) \tau(f)$ is zero.

In order to prove the second equation of assertion (h) note that $\left|\sigma_{T}\right|(d)=\left|\sigma_{T}\right|(c)+\left|\sigma_{T}\right|(a)<$ $2\left|\sigma_{T}\right|(c)<\left|\sigma_{T}\right|(b)$. Thus (e) gives that

$$
\begin{aligned}
& \Delta(\iota(\tau(d)))-\iota(\tau(d)) \otimes 1-g(d)^{-1} \otimes \iota(\tau(d))-\lambda(d) g(a)^{-1} \iota(\tau(c)) \otimes \iota(\tau(a)) \\
& \quad \in\left(\iota\left(S\left(\gamma\left(c_{\mathrm{R}}\right)\right)^{+}+\tau(c) S\left(\gamma\left(c_{\mathrm{R}}\right)\right)^{+}\right) \# k G\right) \otimes \mathcal{B}\left(V^{*}\right) .
\end{aligned}
$$

By this fact and assumption (f) one obtains that

$$
\begin{aligned}
\left\langle\iota(\tau(d)), \tau(b) \tau(a)-\chi(b, a) \tau(a) \tau(b)-\frac{\lambda(b) \lambda\left(c_{\mathrm{R}}\right)}{(3)_{p_{c}}^{!}} \tau(c)^{3}\right\rangle \\
=\langle\iota(\tau(d)), \tau(b)\rangle \tau(a)+\lambda(d)\left\langle g(a)^{-1} \iota(\tau(c)), \tau(b)\right\rangle\langle\iota(\tau(a)), \tau(a)\rangle \\
\quad-\chi(b, a) \chi(d, a)^{-1} \tau(a)\langle\iota(\tau(d)), \tau(b)\rangle \\
\quad-\frac{\lambda(b) \lambda\left(c_{\mathrm{R}}\right)}{(3)_{p_{c}}^{!}}\left[\chi(d, c)^{-1} \tau(c)\left\langle\iota(\tau(d)), \tau(c)^{2}\right\rangle+\lambda(d)\langle\iota(\tau(c)), \tau(c)\rangle\left\langle\iota(\tau(a)), \tau(c)^{2}\right\rangle\right] .
\end{aligned}
$$

Using Lemma 8.2(iii) and arguments as in the previous case one obtains the required result. This completes the proof of Proposition 8.1.

Proof of Theorem 7.1. The proof is based on Corollary 8.1 and consists of a case by case checking of admissibility of $(T, V, n)$ for all $n \in \mathbb{N}$. The computations are elementary, but they still need a 


\section{HECKENBERGER}

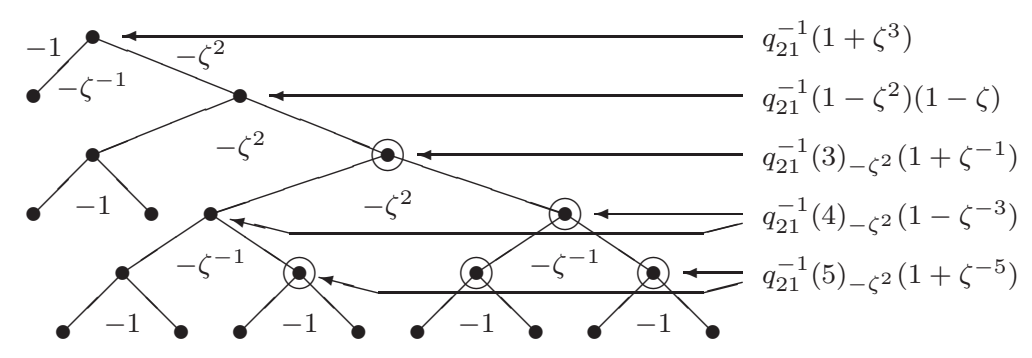

Figure 5. The example with tree T22.

considerable amount of time. However, in order to prove the first condition of admissibility, it is possible to avoid the explicit computation of the $\lambda(a)$, where $a \in N_{2}(T)$, as follows.

Suppose that $a \in N_{2}(T)$. Let $b, c \in N_{0}(T)$ be the unique nodes such that $b^{\mathrm{R}}=c^{\mathrm{L}}=a$. Then by (8) one obtains that

$$
\begin{aligned}
& \lambda(b)=\lambda(a)+\left(\chi\left(a^{\mathrm{L}}, a\right)^{-1}-\chi\left(a, b^{\mathrm{L}}\right)\right)\left(\ell^{\mathrm{L}}(b)\right)_{p_{a}}, \\
& \lambda(c)=\lambda(a)+\left(\chi\left(a, a^{\mathrm{R}}\right)^{-1}-\chi\left(c^{\mathrm{R}}, a\right)\right)\left(\ell^{\mathrm{R}}(c)\right)_{p_{a}} .
\end{aligned}
$$

Thus one gets

$$
\begin{aligned}
\lambda(b) & =\lambda(c) \\
& \Leftrightarrow \chi\left(a^{\mathrm{L}}, a\right)^{-1}\left(\ell^{\mathrm{L}}(b)\right)_{p_{a}}+\chi\left(c^{\mathrm{R}}, a\right)\left(\ell^{\mathrm{R}}(c)\right)_{p_{a}}=\chi\left(a, b^{\mathrm{L}}\right)\left(\ell^{\mathrm{L}}(b)\right)_{p_{a}}+\chi\left(a, a^{\mathrm{R}}\right)^{-1}\left(\ell^{\mathrm{R}}(c)\right)_{p_{a}} \\
& \Leftrightarrow\left(\ell^{\mathrm{L}}(b)+\ell^{\mathrm{R}}(c)\right)_{p_{a}}\left(\chi\left(c^{\mathrm{R}}, a\right)-\chi\left(a, b^{\mathrm{L}}\right)\right)=0 \\
& \Leftrightarrow\left(\ell^{\mathrm{L}}(b)+\ell^{\mathrm{R}}(c)\right)_{p_{a}}\left(p_{a^{\mathrm{R}}}-p_{a}^{\ell^{\mathrm{L}}(b)-\ell^{\mathrm{R}}(c)} p_{a^{\mathrm{L}}}\right)=0 .
\end{aligned}
$$

Further, if $a \in N(T)$ and $a^{\mathrm{R}}=$ ' $R$ ' then

$$
\lambda(a)=q_{21}^{-1}\left(1-q_{11}^{\ell^{\mathrm{L}}(a)-1} q_{12} q_{21}\right)\left(\ell^{\mathrm{L}}(a)\right)_{q_{11}^{-1}}
$$

and if $a \in N(T)$ and $a^{\mathrm{L}}={ }^{\prime} L$ ' then

$$
\lambda(a)=q_{21}^{-1}\left(1-q_{22}^{\ell^{\mathrm{R}}(a)-1} q_{12} q_{21}\right)\left(\ell^{\mathrm{R}}(a)\right)_{q_{22}^{-1}} .
$$

Equations (12), (13), and (14) give an effective method to check the first condition of admissibility of $(T, V, n)$ for all $n$. In fact, the equivalence between $\lambda(a)=0$ and $a \in N_{0}(T)$ holds for all $a \in N(T)$ if and only if

$$
\begin{aligned}
\ell_{\mathrm{R}}(\mathbf{r}) & =\min \left\{m \in \mathbb{N} \mid(m)_{q_{11}^{-1}}\left(q_{11}^{m-1} q_{12} q_{21}-1\right)=0\right\}, \\
\ell_{\mathrm{L}}(\mathbf{r}) & =\min \left\{m \in \mathbb{N} \mid(m)_{q_{22}^{-1}}\left(q_{22}^{m-1} q_{12} q_{21}-1\right)=0\right\}, \\
\ell_{\mathrm{R}}\left(a_{\mathrm{L}}\right) & =\min \left\{m \in \mathbb{N} \mid\left(m+\ell_{\mathrm{L}}\left(a_{\mathrm{R}}\right)\right)_{p_{a}}\left(p_{a^{\mathrm{R}}} p_{a}^{\ell_{\mathrm{L}}\left(a_{\mathrm{R}}\right)}-p_{a^{\mathrm{L}}} p_{a}^{m}\right)=0\right\}
\end{aligned}
$$

for all $a \in N_{2}(T)$.

As an example consider the last entry of Table 1. Thus the generalized Dynkin diagram of $V$ is

$$
\stackrel{-\zeta^{-2}-\zeta^{3}-1}{\longrightarrow}
$$

and $T$ is the tree T22. In Figure 5 the numbers $p_{a}$, where $a \in N_{2}(T)$, are given as a label below the node $a$. Then (15) can be easily checked. Moreover, the second and third conditions of admissibility concerning the values of the $p_{a}$ and the first relation of the fourth condition are also fulfilled by Figure 5. The relation $\ell_{\mathrm{R}}\left(c_{\mathrm{L}}\right) \leqslant \ell^{\mathrm{R}}(b)$ in the fourth condition, where $c=b^{\mathrm{L}}$, is not fulfilled exactly for those nodes $b$ which are marked with a big circle. In this case one can see that $\ell^{\mathrm{R}}(b) \leqslant 2$, 

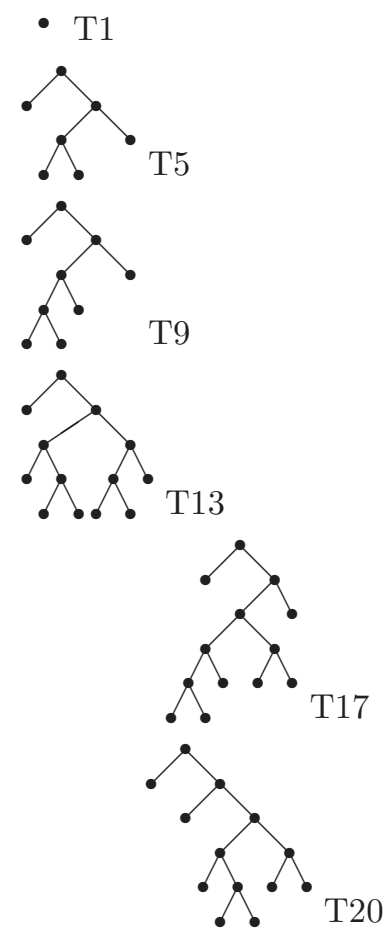

$\triangle \mathrm{T} 2$
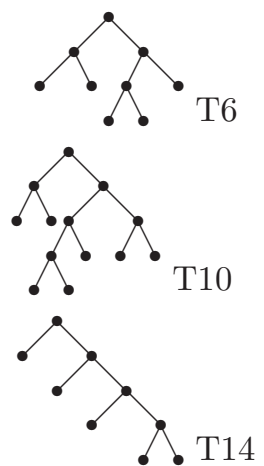
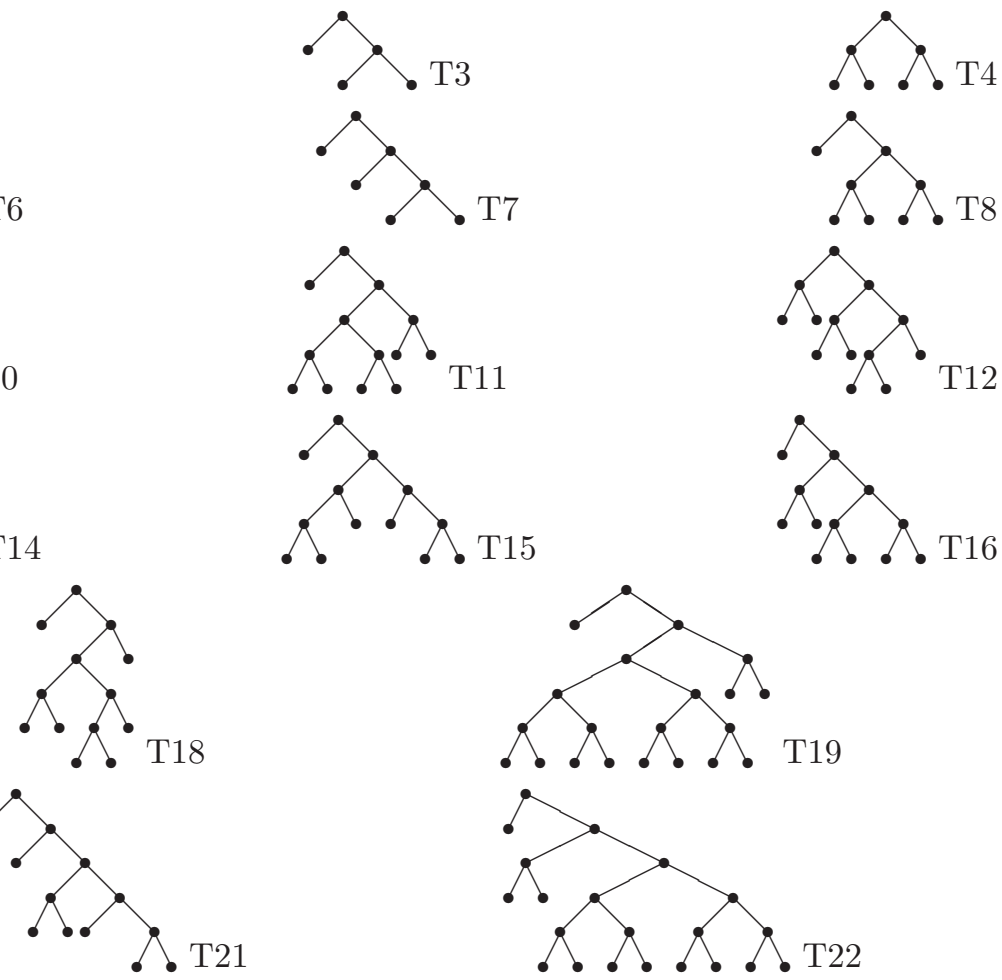

Figure A1.

and for all five nodes the computation of $\nu(b)$, using the values of $\lambda(\cdot)$ noted on the right-hand side of Figure 5, gives 0 .

Some data regarding the other examples can be found in Appendix A. They may be helpful to check the admissibility of the corresponding triples $(T, V, n)$.

\section{ACKNOWLEDGEMENTS}

The author wants to thank A. Joseph and S. Ufer for stimulating discussions and N. Andruskiewitsch and M. Graña for helpful remarks. Many thanks also go to the referee for his very useful hints concerning mainly the legibility of the paper.

\section{Appendix A. Types of Nichols algebras}

In Figure A1 we collect all full binary trees which appear as the type of some Nichols algebra of diagonal type studied in the present paper.

Finally, in order to make it easier to check the admissibility of the triples $(T, V, n)$, in each example, the numbers $p_{a}$ are listed below. If for some node $b$ it is necessary to compute $\nu(b)$, the values $\lambda(a)$ involved in the formula will be given, except when $a^{\mathrm{R}}={ }^{\prime} R$ '. In the latter case one can 


\section{HECKENBERGER}

take formula (13). The nodes of the tree $T$ are denoted by $a_{Q}$, where $Q=r / s$ is the value of the function $Q$ defined in (7). We write $p_{Q}$ for $p_{a_{Q}}$. Note that some diagrams are omitted since they can be obtained from another one by choosing other parameters with the same properties.

$$
\begin{aligned}
& \begin{array}{ccc}
q & r & q, r \in k^{*}, \mathrm{~T} 1: p_{0}=r^{-1}, p_{\infty}=q^{-1} .
\end{array}
\end{aligned}
$$

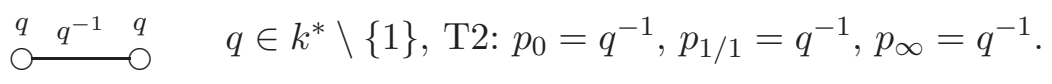

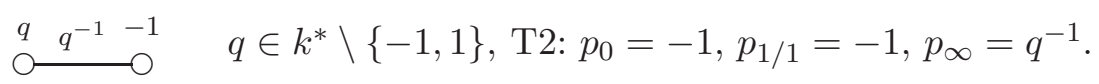

$$
\begin{aligned}
& \stackrel{-1}{\circ} \underset{\sim}{\stackrel{-1}{\longrightarrow}} q q k^{*} \backslash\{-1,1\}, \mathrm{T} 2: p_{0}=-1, p_{1 / 1}=q^{-1}, p_{\infty}=-1 .
\end{aligned}
$$

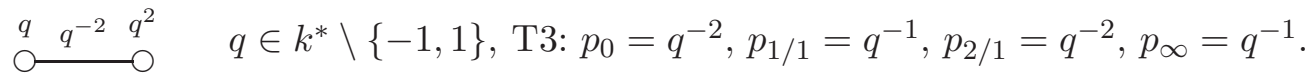

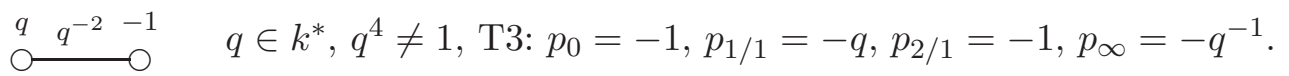

$$
\begin{aligned}
& {\stackrel{\zeta}{q^{-1}}}^{q} \quad \zeta \in R_{3}, q \in k^{*}, q^{3} \neq 1, \mathrm{~T} 3: p_{0}=q^{-1}, p_{1 / 1}=\zeta^{-1}, p_{2 / 1}=\zeta^{-1} q, p_{\infty}=\zeta^{-1} \text {. }
\end{aligned}
$$

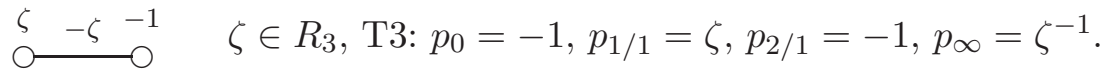

$$
\begin{aligned}
& \stackrel{-\zeta^{-2}-\zeta^{3}-\zeta^{2}}{\mathrm{O}} \mathrm{C} \quad \zeta \in R_{12}, \mathrm{~T} 4: p_{0}=-\zeta^{-2}, p_{1 / 2}=-1, p_{1 / 1}=\zeta^{3}, p_{2 / 1}=-1, p_{\infty}=-\zeta^{2} . \\
& \stackrel{-\zeta^{2}-\zeta^{-1}}{\mathrm{O}} \quad \zeta \in R_{12}, \mathrm{~T} 5: p_{0}=-1, p_{1 / 1}=\zeta^{3}, p_{3 / 2}=-1, p_{2 / 1}=-\zeta^{2}, p_{\infty}=-\zeta^{-2} .
\end{aligned}
$$

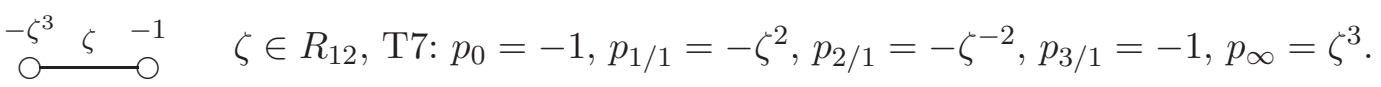

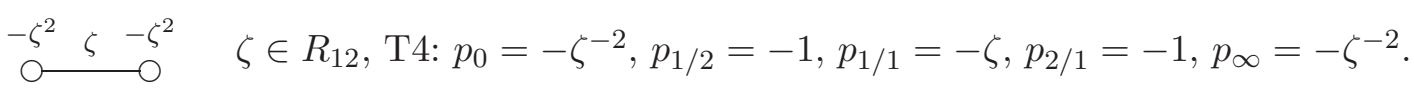

$$
\begin{aligned}
& \underset{-\zeta^{2} \zeta^{3}-1}{\mathrm{O}} \mathrm{C} \quad \zeta \in R_{12}, \mathrm{~T} 5: p_{0}=-1, p_{1 / 1}=-\zeta, p_{3 / 2}=-1, p_{2 / 1}=-\zeta^{-2}, p_{\infty}=-\zeta^{-2} . \\
& \stackrel{-\zeta^{-1}-\zeta^{3}-1}{\mathrm{O}} \mathrm{O} \quad \zeta \in R_{12}, \mathrm{~T} 7: p_{0}=-1, p_{1 / 1}=-\zeta^{-2}, p_{2 / 1}=-\zeta^{-2}, p_{3 / 1}=-1, p_{\infty}=-\zeta . \\
& \stackrel{-\zeta \zeta^{-2} \zeta^{3}}{\circ} \quad \zeta \in R_{9}, \mathrm{~T} 6: p_{0}=\zeta^{-3}, p_{1 / 2}=-1, p_{1 / 1}=-\zeta^{-2}, p_{3 / 2}=-1, p_{2 / 1}=\zeta^{-3}, p_{\infty}=-\zeta^{-1} . \\
& \stackrel{\zeta^{3}}{\zeta^{-1}-1} \underset{\longrightarrow}{\longrightarrow} \zeta \in R_{9}, \mathrm{~T} 9: p_{0}=-1, p_{1 / 1}=-\zeta^{-2}, p_{4 / 3}=-1, p_{3 / 2}=\zeta^{-3}, p_{2 / 1}=-\zeta^{-1}, p_{\infty}=\zeta^{-3} .
\end{aligned}
$$

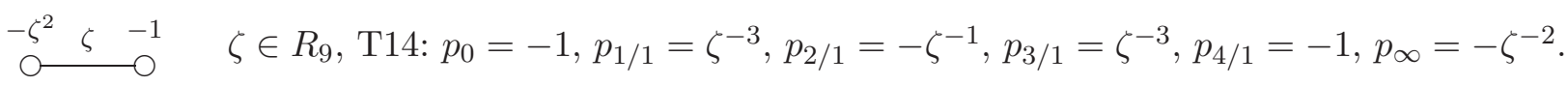

$$
\begin{aligned}
& \stackrel{q}{\mathrm{q}} \stackrel{q^{-3}}{{ }^{3}} \stackrel{q}{\longrightarrow} \quad q \in k^{*}, q^{2}, q^{3} \neq 1, \mathrm{~T} 8: p_{0}=q^{-3}, p_{1 / 1}=q^{-1}, p_{3 / 2}=q^{-3}, p_{2 / 1}=q^{-1}, \\
& p_{3 / 1}=q^{-3}, p_{\infty}=q^{-1} \text {. } \\
& \stackrel{\zeta^{2}}{\zeta \zeta^{-1}} \underset{\longrightarrow}{\longrightarrow} \zeta \in R_{8}, \mathrm{~T} 8: p_{0}=\zeta, p_{1 / 1}=-\zeta^{2}, p_{3 / 2}=-1, p_{2 / 1}=\zeta^{-1}, p_{3 / 1}=-1, p_{\infty}=-\zeta^{2} . \\
& \stackrel{\zeta^{2}-\zeta^{-1}-1}{\longrightarrow} \quad \zeta \in R_{8}, \mathrm{~T} 8: p_{0}=-1, p_{1 / 1}=\zeta^{-1}, p_{3 / 2}=-1, p_{2 / 1}=-\zeta^{2}, p_{3 / 1}=\zeta, p_{\infty}=-\zeta^{2} \text {. }
\end{aligned}
$$




\section{RANK 2 Nichols ALGEBRAS OF DIAGONAL TYPE}

$\stackrel{\zeta}{\zeta}-{ }^{-}-{ }^{-1}$

$\stackrel{\zeta^{6}-\zeta^{-1-}-\zeta^{-4}}{\longrightarrow}$

$\stackrel{\zeta^{6} \quad \zeta \quad \zeta^{-1}}{\circ}$

$\stackrel{\zeta^{-4} \zeta^{5}}{\longrightarrow} \mathrm{\longrightarrow}^{-1}$

$\stackrel{\zeta}{\zeta} \zeta^{-5}-1$

$\stackrel{\zeta}{\zeta} \zeta^{2} \quad-1$

$\stackrel{\zeta^{-2} \zeta^{-2}-1}{\longrightarrow}$

$\stackrel{\zeta}{\lessgtr} \zeta^{-3}-1$

$\stackrel{-\zeta^{-2} \zeta^{3}}{\circ}{ }^{-1}$

$\stackrel{-\zeta-\zeta^{-3} \zeta^{5}}{ }$

$\overbrace{}^{3}-\zeta^{4-\zeta^{-4}}$

$\stackrel{\zeta^{5}-\zeta^{-2}-1}{\longrightarrow}$

$\stackrel{\zeta^{3}-\zeta^{2}-1}{\longrightarrow}$

$\stackrel{-\zeta-\zeta^{-3}-1}{\longrightarrow}$

$\zeta \in R_{8}, \mathrm{~T} 8: p_{0}=-1, p_{1 / 1}=-\zeta^{2}, p_{3 / 2}=\zeta, p_{2 / 1}=-\zeta^{2}, p_{3 / 1}=-1, p_{\infty}=\zeta^{-1}$.

$\zeta \in R_{24}, \mathrm{~T} 10: p_{0}=-\zeta^{4}, p_{1 / 2}=-1, p_{1 / 1}=\zeta^{-1}, p_{4 / 3}=-1, p_{3 / 2}=-\zeta^{4}, p_{2 / 1}=-\zeta^{6}$, $p_{3 / 1}=\zeta, p_{\infty}=-\zeta^{6}$.

$\zeta \in R_{24}, \mathrm{~T} 13: p_{0}=\zeta, p_{1 / 1}=-\zeta^{6}, p_{3 / 2}=-\zeta^{4}, p_{5 / 3}=-1, p_{2 / 1}=\zeta^{-1}, p_{5 / 2}=-1$, $p_{3 / 1}=-\zeta^{4}, p_{\infty}=-\zeta^{6}$.

$\zeta \in R_{24}, \mathrm{~T} 17: p_{0}=-1, p_{1 / 1}=\zeta^{-1}, p_{5 / 4}=-1, p_{4 / 3}=-\zeta^{4}, p_{3 / 2}=-\zeta^{6}, p_{5 / 3}=\zeta$, $p_{2 / 1}=-\zeta^{6}, p_{\infty}=-\zeta^{4}, \lambda\left(a_{3 / 2}\right)=q_{21}^{-1}\left(1+\zeta+\zeta^{2}\right)\left(1-\zeta^{3}\right), \lambda\left(a_{5 / 3}\right)=q_{21}^{-1} \zeta^{2}(1-\zeta)$.

$\zeta \in R_{24}, \mathrm{~T} 21: p_{0}=-1, p_{1 / 1}=-\zeta^{4}, p_{2 / 1}=-\zeta^{6}, p_{5 / 2}=\zeta, p_{3 / 1}=-\zeta^{6}, p_{4 / 1}=-\zeta^{4}$, $p_{5 / 1}=-1, p_{\infty}=\zeta^{-1}$.

$\zeta \in R_{5}$, T11: $p_{0}=-1, p_{1 / 1}=-\zeta^{2}, p_{4 / 3}=-1, p_{3 / 2}=\zeta^{-1}, p_{5 / 3}=-1, p_{2 / 1}=-\zeta^{2}$, $p_{3 / 1}=-1, p_{\infty}=\zeta^{-1}, \lambda\left(a_{3 / 2}\right)=q_{21}^{-1}\left(1+\zeta^{-1}\right)\left(1-\zeta^{2}\right), \lambda\left(a_{5 / 3}\right)=q_{21}^{-1}(1-\zeta)\left(1-\zeta^{2}\right)$.

$\zeta \in R_{5}, \mathrm{~T} 16: p_{0}=-1, p_{1 / 1}=\zeta^{-1}, p_{3 / 2}=-1, p_{2 / 1}=-\zeta^{2}, p_{5 / 2}=-1, p_{3 / 1}=\zeta^{-1}$, $p_{4 / 1}=-1, p_{\infty}=-\zeta^{2}$.

$\zeta \in R_{20}, \mathrm{~T} 11: p_{0}=-1, p_{1 / 1}=-\zeta^{2}, p_{4 / 3}=-1, p_{3 / 2}=-\zeta^{-1}, p_{5 / 3}=-1, p_{2 / 1}=-\zeta^{2}$, $p_{3 / 1}=-1, p_{\infty}=\zeta^{-1}, \lambda\left(a_{3 / 2}\right)=q_{21}^{-1}(1-\zeta)\left(1-\zeta^{-2}+\zeta^{-4}\right)$, $\lambda\left(a_{5 / 3}\right)=q_{21}^{-1} \zeta\left(\zeta^{2}-1\right)\left(1+\zeta^{-3}\right)\left(1+\zeta^{-4}\right)$.

$\zeta \in R_{20}, \mathrm{~T} 16: p_{0}=-1, p_{1 / 1}=\zeta^{-1}, p_{3 / 2}=-1, p_{2 / 1}=-\zeta^{2}, p_{5 / 2}=-1, p_{3 / 1}=-\zeta^{-1}$, $p_{4 / 1}=-1, p_{\infty}=-\zeta^{2}$.

$\zeta \in R_{15}, \mathrm{~T} 12: p_{0}=\zeta^{-5}, p_{1 / 2}=-1, p_{1 / 1}=\zeta^{-3}, p_{3 / 2}=-\zeta^{4}, p_{2 / 1}=\zeta^{-3}, p_{5 / 2}=-1$, $p_{3 / 1}=\zeta^{-5}, p_{\infty}=-\zeta^{-1}$.

$\zeta \in R_{15}, \mathrm{~T} 15: p_{0}=-\zeta^{4}, p_{1 / 1}=\zeta^{-3}, p_{4 / 3}=-1, p_{3 / 2}=\zeta^{-5}, p_{2 / 1}=-\zeta^{-1}, p_{3 / 1}=\zeta^{-5}$, $p_{4 / 1}=-1, p_{\infty}=\zeta^{-3}$.

$\zeta \in R_{15}$, T18: $p_{0}=-1, p_{1 / 1}=\zeta^{-3}, p_{4 / 3}=-\zeta^{4}, p_{3 / 2}=\zeta^{-3}, p_{8 / 5}=-1, p_{5 / 3}=\zeta^{-5}$, $p_{2 / 1}=-\zeta^{-1}, p_{\infty}=\zeta^{-5}, \lambda\left(a_{3 / 2}\right)=q_{21}^{-1}\left(1+\zeta^{3}+\zeta^{6}\right)\left(1+\zeta^{-8}\right)$, $\lambda\left(a_{5 / 3}\right)=q_{21}^{-1} \zeta\left(1+\zeta^{3}\right)\left(1+\zeta^{-5}\right)$.

$\zeta \in R_{15}$, T20: $p_{0}=-1, p_{1 / 1}=\zeta^{-5}, p_{2 / 1}=-\zeta^{-1}, p_{5 / 2}=\zeta^{-5}, p_{8 / 3}=-1, p_{3 / 1}=\zeta^{-3}$, $p_{4 / 1}=-\zeta^{4}, p_{\infty}=\zeta^{-3}$.

$\zeta \in R_{7}, \mathrm{~T} 19: p_{0}=-1, p_{1 / 1}=-\zeta^{2}, p_{5 / 4}=-1, p_{4 / 3}=-\zeta^{-1}, p_{7 / 5}=-1, p_{3 / 2}=-\zeta^{2}$, $p_{8 / 5}=-1, p_{5 / 3}=-\zeta^{-1}, p_{7 / 4}=-1, p_{2 / 1}=-\zeta^{2}, p_{3 / 1}=-1, p_{\infty}=\zeta^{-1}$, $\lambda\left(a_{3 / 2}\right)=q_{21}^{-1}\left(1+\zeta^{-1}\right)\left(\zeta-\zeta^{-1}+\zeta^{-3}\right)$, $\lambda\left(a_{4 / 3}\right)=\lambda\left(a_{5 / 3}\right)=q_{21}^{-1}\left(1-\zeta^{-1}\right)\left(1-\zeta^{-2}\right), \lambda\left(a_{7 / 5}\right)=\lambda\left(a_{7 / 4}\right)=q_{21}^{-1}\left(1+\zeta^{-3}\right)$.

$\stackrel{-\zeta^{-2}-\zeta^{3}-1}{\longrightarrow} \quad \zeta \in R_{7}$, T22: see Figure 5. 


\section{RANK 2 Nichols ALGEBRAS OF DiAgONAL TYPE}

\section{REFERENCES}

And02 N. Andruskiewitsch, About finite dimensional Hopf algebras, in Quantum symmetries in theoretical physics and mathematics, Bariloche, 2000, Contemporary Mathematics, vol. 294 (American Mathematical Society, Providence, RI, 2002), 1-57.

And04 N. Andruskiewitsch, Some remarks on Nichols algebras, in Hopf algebras, DePaul University, 2003, Lecture Notes in Pure and Applied Mathematics, vol. 237 (Dekker, New York, 2004), 35-45.

AG99 N. Andruskiewitsch and M. Graña, Braided Hopf algebras over non-abelian finite groups, Bol. Acad. Nac. Cienc. (Córdoba) 63 (1999), 45-78.

AS98 N. Andruskiewitsch and H.-J. Schneider, Lifting of quantum linear spaces and pointed Hopf algebras of order $p^{3}$, J. Algebra 209 (1998), 658-691.

AS00 M. Andruskiewitsch and H.-J. Schneider, Finite quantum groups and Cartan matrices, Adv. Math. 154 (2000), 1-45.

AS02 N. Andruskiewitsch and H.-J. Schneider, Pointed Hopf algebras, in New Directions in Hopf Algebras, Mathematical Sciences Research Institute Publications, vol. 43 (Cambridge University Press, 2002).

CLR90 T. H. Cormen, C. E. Leiserson and R. L. Rivest, Introduction to algorithms (MIT Press, Cambridge, MA, 1990).

GKP94 R. L. Graham, D. E. Knuth and O. Patashnik, Concrete mathematics: a foundation for computer science (Addison-Wesley, Reading, MA, 1994).

Gra00a M. Graña, A freeness theorem for Nichols algebras, J. Algebra 231 (2000), 235-257.

Gra00b M. Graña, On Nichols algebras of low dimension, in New trends in Hopf algebra theory, La Falda, 1999, Contemporary Mathematics, vol. 267 (American Mathematical Society, Providence, RI, 2000), 111-134.

Hec04 I. Heckenberger, Rank 2 Nichols algebras with finite arithmetic root system, Preprint (2004), math.QA/0412458.

Hec06 I. Heckenberger, The Weyl groupoid of a Nichols algebra of diagonal type, Invent. Math. 164 (2006), $175-188$.

Kha99 V. Kharchenko, A quantum analog of the Poincaré-Birkhoff-Witt theorem, Algebra Logic 38 (1999), 259-276.

Lot83 M. Lothaire, Combinatorics on words (Cambridge University Press, Cambridge, 1983).

Lus93 G. Lusztig, Introduction to quantum groups (Birkhäuser, Boston, 1993).

Nic78 W. D. Nichols, Bialgebras of type one, Comm. Algebra 6 (1978), 1521-1552.

Ros98 M. Rosso, Quantum groups and quantum shuffles, Invent. Math. 133 (1998), 399-416.

Sch96 P. Schauenburg, A characterization of the Borel-like subalgebras of quantum enveloping algebras, Comm. Algebra 24 (1996), 2811-2823.

Ste58 M. A. Stern, Über eine zahlentheoretische Funktion, J. reine angew. Math. 55 (1858), 193-220.

Ufe04 S. Ufer, PBW bases for a class of braided Hopf algebras, J. Algebra 280 (2004), 84-119.

Wor89 S. L. Woronowicz, Differential calculus on compact matrix pseudogroups (quantum groups), Comm. Math. Phys. 122 (1989), 125-170.

I. Heckenberger istvan.heckenberger@math.uni-leipzig.de

Universität Leipzig, Mathematisches Institut, Augustusplatz 10-11, 04109 Leipzig, Germany 